Supporting Information for:

\title{
Origin of Low Temperature Trion Emission in CdSe Nanoplatelets
}

Albert F. Vong ${ }^{a}$, Shawn Irgen-Gioro ${ }^{b}$, Yue Wu ${ }^{b}$, and Emily A. Weiss $* a, b$

${ }^{a}$ Department of Materials Science and Engineering, Northwestern University, 2145 Sheridan Rd., Evanston, IL 60208-3113

${ }^{b}$ Department of Chemistry, Northwestern University, 2145 Sheridan Rd., Evanston, IL 60208-3113

*corresponding author. Email: e-weiss@northwestern.edu

\section{Experimental Methods}

Nanoplatelet synthesis. The CdSe NPLs and CdSe/CdS core/shell NPLs were synthesized following published procedures with minor modifications ${ }^{1} .170 \mathrm{mg}$ freshly prepared cadmium myristate (Cd(myr) $\left.)_{2}\right), 12 \mathrm{mg}$ selenium powder (99.99\% trace metals basis) and $5 \mathrm{~mL} 1$-Octadecene (ODE) were added in a 50-mL three-neck flask and degassed under vacuum for one hour at $90{ }^{\circ} \mathrm{C}$. After degassing the mixture was heated to $240{ }^{\circ} \mathrm{C}$ under Argon flow. When the temperature reached $190^{\circ} \mathrm{C}, 40 \mathrm{mg}$ cadmium acetate $\left(\mathrm{Cd}(\mathrm{OAc})_{2} \cdot 2 \mathrm{H}_{2} \mathrm{O}, 98 \%\right)$ was swiftly added into the flask. The reaction remained at $240{ }^{\circ} \mathrm{C}$ for $10 \mathrm{~min}$ before cooling down. $1.5 \mathrm{~mL}$ oleic acid and $15 \mathrm{~mL}$ hexane were added at $80{ }^{\circ} \mathrm{C}$ to stabilize the NPLs. The solution was then centrifuged at $7500 \mathrm{rpm}$ for $5 \mathrm{~min}$ and the pellets were dissolved in hexane. To prepare the precursor $\mathrm{Cd}(\mathrm{myr})_{2}, 120 \mathrm{mg}$ ( 3 mmol) $\mathrm{NaOH}$ and $685.2 \mathrm{mg}(3 \mathrm{mmol})$ myristic acid were sequentially dissolved in $20 \mathrm{~mL}$ methanol. After the mixture was fully dissolved, $10 \mathrm{~mL} 0.1 \mathrm{M}$ cadmium nitrate $\left(\mathrm{Cd}\left(\mathrm{NO}_{3}\right)_{2}\right)$ methanol solution was added dropwise under stirring. After 30 min the suspension was vacuum filtered. The product was dried in vacuum oven overnight before use. Transmission electron microscope (TEM) and Scanning transmission electron microscopy (STEM) images for the 4ML core-only NPLs and corresponding size distributions are shown in Figure S1. 
a

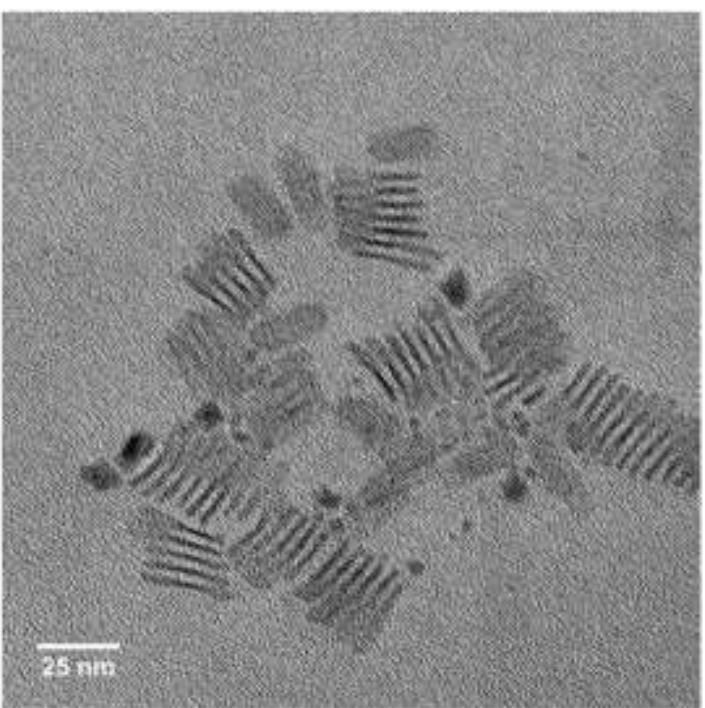

b

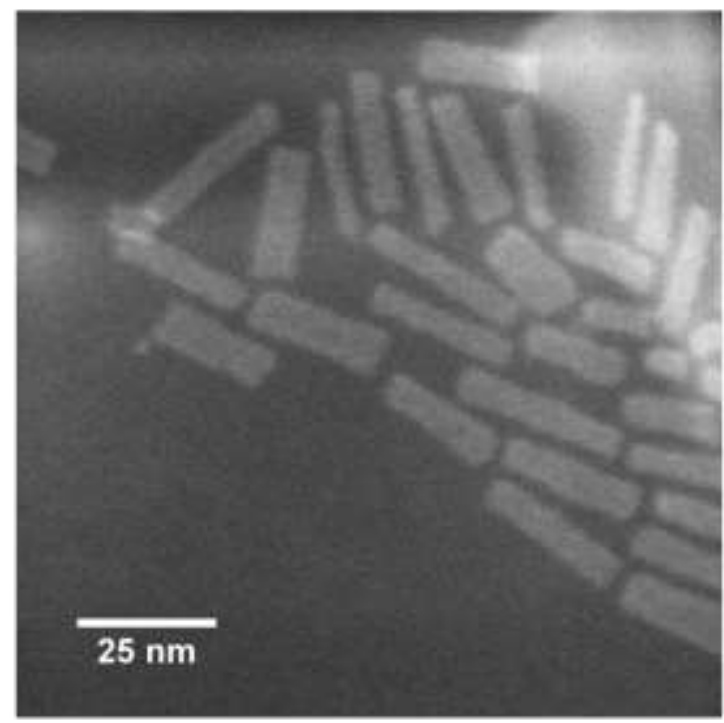

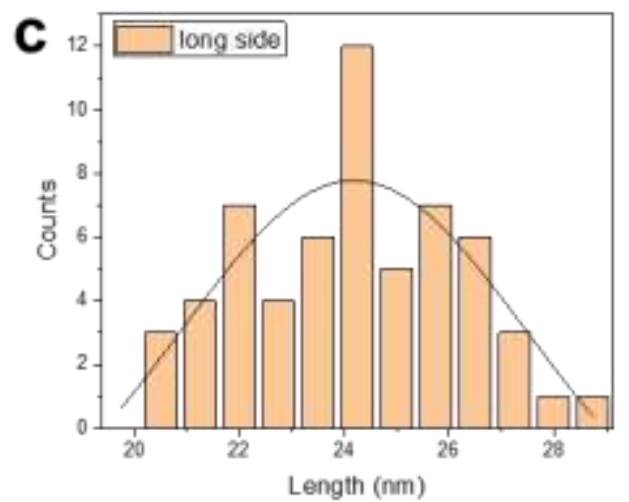

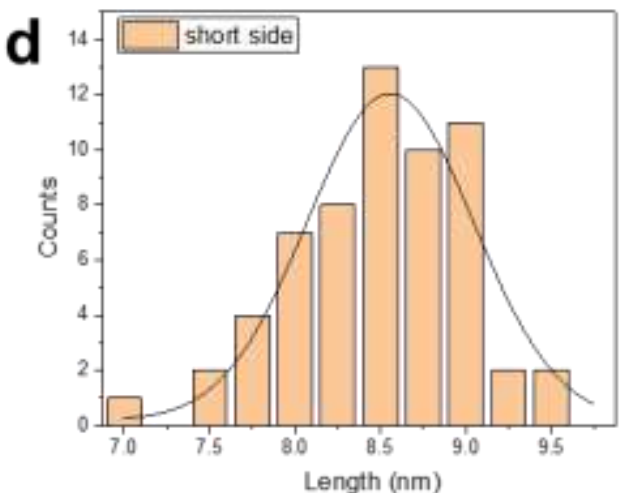

e

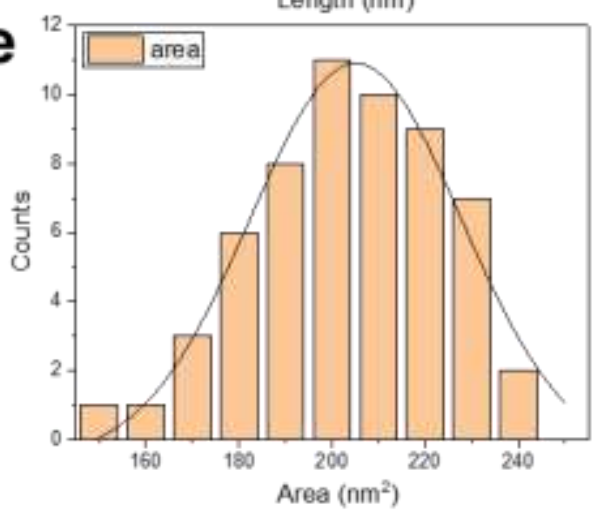

Figure S1. (a) TEM Image of 4ML CdSe core-only NPLs. (b) STEM image of 4ML CdSe Coreonly NPLs. (c,d,e) Histograms of the short side length, long side length, and area of the NPLs, respectively, yielding an average size of $24.2 \pm 3.3 \mathrm{~nm} \times 8.6 \pm 0.5 \mathrm{~nm}\left(205 \mathrm{~nm}^{2} \pm 20.2 \mathrm{~nm}^{2}\right)$. These were generated from size analysis on TEM images. 
Film Deposition Details. This method was adapted from published procedures ${ }^{2}$. As-prepared 4.5 ML CdSe NPLs were suspended in hexanes. 40-50 $\mu \mathrm{L}$ of the CdSe solution was slowly delivered to the side of a $25-\mathrm{mL}$, round PTFE crucible containing $5 \mathrm{~mL}$ of dried acetonitrile and a glass substrate submerged in the center, which was placed on an elevated PTFE platform. The CdSe NPLs formed a growing sheet of a relatively consistent monolayer that completely covered the subphase surface; this process would take around 15-20 seconds. The concentration of the CdSe solution was adjusted based on the amount of surface coverage required to achieve a relatively uniform CdSe layer. We allowed hexanes at top interface to completely evaporate for several minutes, leaving behind the NPL layer on lying on acetonitrile, which serves as an antisolvent for the NPLs. The antisolvent was slowly drained using a pipette, until the overlying CdSe layer was deposited on the substrate surface. The remaining acetonitrile subphase was allowed to evaporate over several minutes, until the monolayer had completely settled on the substrate. This deposition method produced much more uniform films versus drop casting or spin coating, which helped with film replicability and uniformity for low temperature TA measurements.

Film thickness was measured with a profilometer. A clean substrate surface was produced via a q-tip soaked in hexanes, producing a measurable "step" from the substrate to nanoplatelet surface (Figure S2).

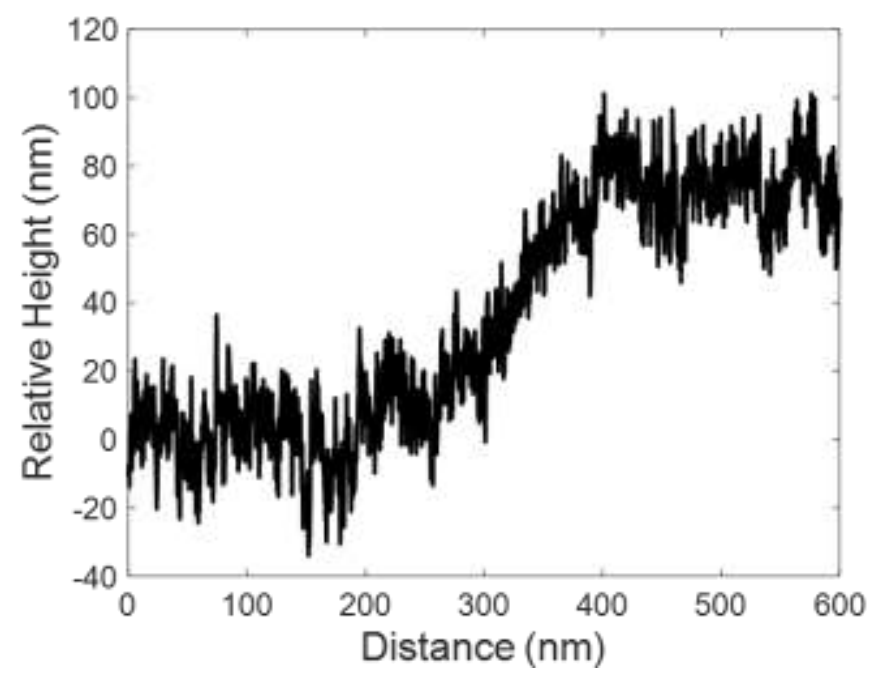

Figure S2. Step profile of CdSe film deposited via antisolvent method. An approximate thickness of $80 \mathrm{~nm}$ was determined from the measurement. 
Diluted and drop-cast film synthesis for excimer characterization. Adapting an isolated NPL film method from Antolinez et al. and Irgen-Gioro, Wu et al., we performed a serial dilution on as-synthesized NPLs and measured the corresponding PL of drop-casted films with these dilutions ${ }^{3,4}$. At an appropriate concentration, we were able to locate isolated emitters and measure their spectra. We obtain similar results as Antolinez et al., namely that undiluted dropcast films have a third peak further red-shifted from the trion $(\sim 2.47 \mathrm{eV}$ in our film) due to excimer emission from nanoplatelet stacking. The diluted NPL spectrum (Figure S3b) shows conclusively that, in the dilute particle limit, there are only two individual emission peaks, matching the energies of the peaks in our layer-by-layer NPL film; this result rules out excimer emission as the source of the $\sim 2.49 \mathrm{eV}$ peak, in agreement with other studies that control for stacking $^{3,5}$.
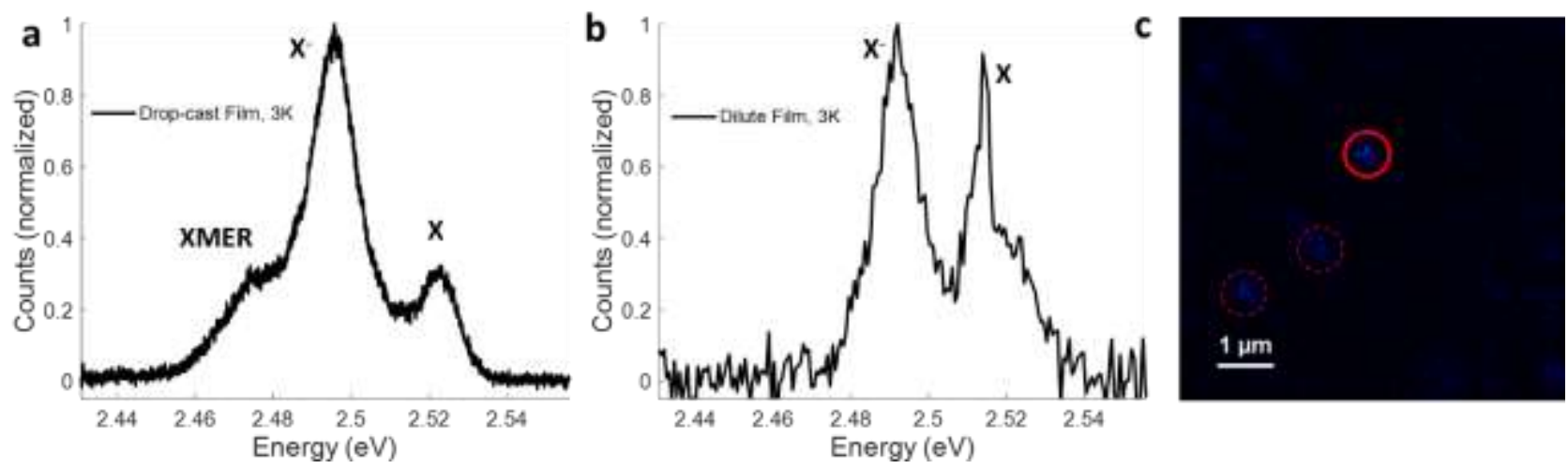

Figure S3. Low temperature spectra of thick NPL films and dilute NPLs at 3 K. (a) PL spectrum of a concentrated, drop-cast NPL film. Besides the trion $\left(\mathrm{X}^{-}\right)$and exciton $(\mathrm{X})$ peaks, there is a third peak attributed to NPL stacking-induced excimer emission (labelled XMER). (b) PL spectrum of diluted emitters indicated with a solid red circle from S3c. The peak attributed to NPL stacking is not present. (c) Photoluminescence map (at $3 \mathrm{~K}$ ) of a diluted NPL film with emitting regions indicated by red circles. 
Variable Temperature Photoluminescence Measurements (PL). Variable temperature PL measurements were taken in a closed-cycle Helium cryostat (Montana Instruments). The film sample was mounted on a piezo positioning system (Attocube, ANC350) in a confocal microscope geometry. To excite the NPLs, a 450-nm laser (PicoQuant LDH-P-C-450B) was directed to the sample using a dichroic beam splitter and a 0.9NA objective.

Sample emission is directed through the same dichroic beamsplitter into an imaging spectrometer (Andor, Shamrock SR-303i-B). A 480-nm long pass filter was used to filter out outgoing excitation light. A grating of 300 lines/mm dispersed emitted light onto an air-cooled electron-multiplying charge-coupled device camera (EMCCD Andor Zyla 4.2P).

Variable Temperature Absorption Measurements. Variable temperature absorption measurements were taken in the same setup described above. The sample was placed in a transmission geometry which allowed light to pass through the sample instead (without the objective used in PL). Instead of a laser excitation source, a broadband incandescence light source was used to illuminate the sample. Transmitted light was measured using the same spectrometer setup as described above.

Doping Photoluminescence Measurements and Excitation Spectra. Photoluminescence measurements of CdSe NPLs with added Bis(pentamethylcyclopentadienyl)cobalt(II) (DMC) were acquired with a Flurolog-3 spectrofluorometer (Horiba Jobin Yvon). Air-free samples of NPL and DMC were prepared in NMR tubes, and placed in a special liquid nitrogen holder. Samples were cooled to liquid nitrogen temperature and allowed to stabilize for 2 minutes, before any measurements were taken. The same geometry was used for obtaining excitation spectra (Figure S6). Excitation spectra was collected at an emission energy of $2.38 \mathrm{eV}$, which is predominantly trion in the emission spectrum (Figure 1c in the main text).

Transient Absorption. Our transient absorption system is described in detail elsewhere ${ }^{6}$. Femtosecond-transient absorption (TA) spectroscopy was performed with a commercial spectrometer (Ultrafast Systems LLC, Helios) and a Ti:sapphire amplifier (Spectra-Physics, Solstice). The fundamental $800 \mathrm{~nm}, 2.5 \mathrm{~mJ}, 100 \mathrm{fs}$ pulse was split to generate pump and probe beams. The pump beam was guided to an optical parametric amplifier (Light Conversion, TOPASC) to generate pump pulses at a wavelength of $358 \mathrm{~nm}$. The white light supercontinuum probe was generated through a 50:50 $\mathrm{H}_{2} \mathrm{O}: \mathrm{D}_{2} \mathrm{O}$ mixture in a 5-mm quartz cuvette. The pump and probe beams 
were overlapped and focused onto the sample film with a repetition rate of $500 \mathrm{~Hz}$ from an optical chopper.

A Janis VNF-100 nitrogen-cooled cryostat was used to house the NPL film samples for TA measurements. The film was prepared on a glass coverslip and centered in the sample chamber, which was placed at the center of the optical transmission window in the cryostat. This setup was also used to measure the spot size of the beam accounting for the optical windows. The sample chamber was cooled down to $85 \mathrm{~K}$ and allowed to stabilize for 30 minutes before measurement.

Doped Films for Absorption Measurements. Doped films for absorption measurements at 3 $\mathrm{K}$ were obained by mixing equivalents of DMC and NPLs in a 2-wt\% polystyrene chloroform solution, adapted from literature procedure ${ }^{7}$. This solution was drop-cast in a nitrogen glovebox to prevent DMC oxidation, and was brought to the cryostat in a sealed container before being quickly mounted onto the stage before pulling vacuum. Doped films scattered additional light, reducing signal to noise in absorption measurements.

Data Analysis. All fitting and plotting was done with home-built MATLAB code.

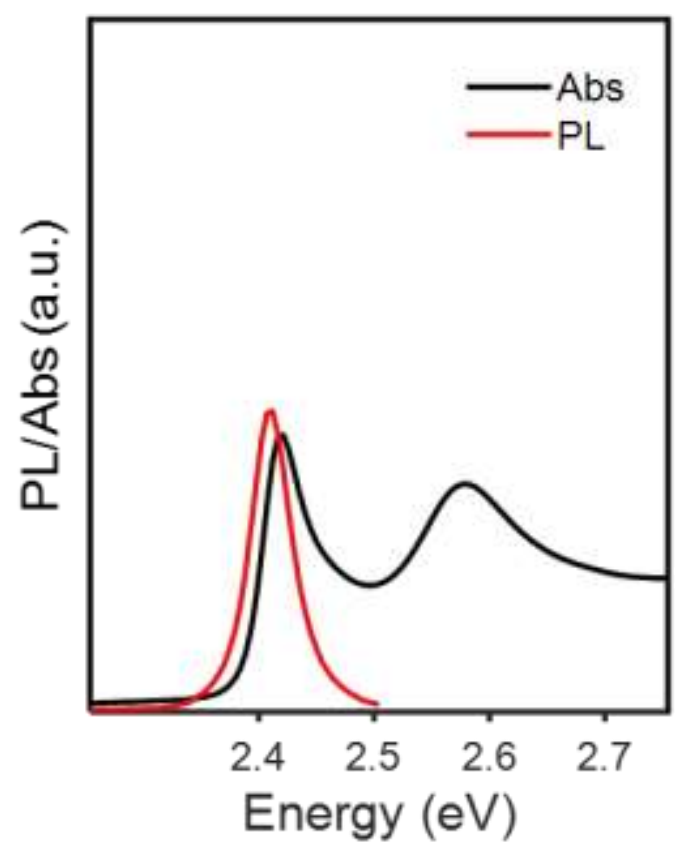

Figure S4. Linear absorption and photoluminescence specra of NPL films at room temperature. There are no collective effects resulting in a red-shifted second emission peak like the trion peak at low temperature. 


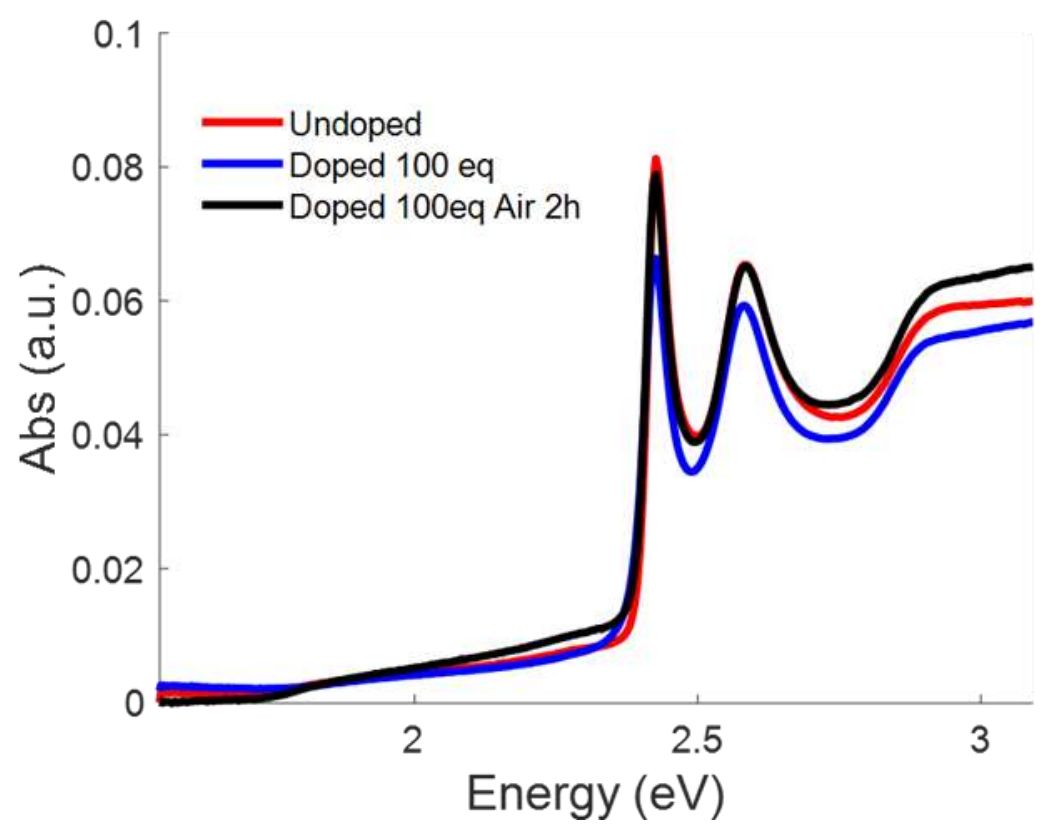

Figure S5. Doped and undoped NPL solution linear absorption spectrum at room temperature. Two cuvettes with identical NPL concentrations were used for this experiment. 100 eq of DMC dopant was added to one cuvette in air-free conditions, and both cuvettes of doped (blue) and undoped NPLs (black) were measured after 10 minutes. A reduction in band-edge absorption for the NPLs with DMC added is indicative of band-edge filling due to n-doping. The n-doped NPLs were then exposed to air for one hour, oxidizing the NPLs and reversing the ndoping process. The air-exposed NPLs (black) largely recover the band-edge absorption, indicating that the $\mathrm{n}$-doping process is reversible. 


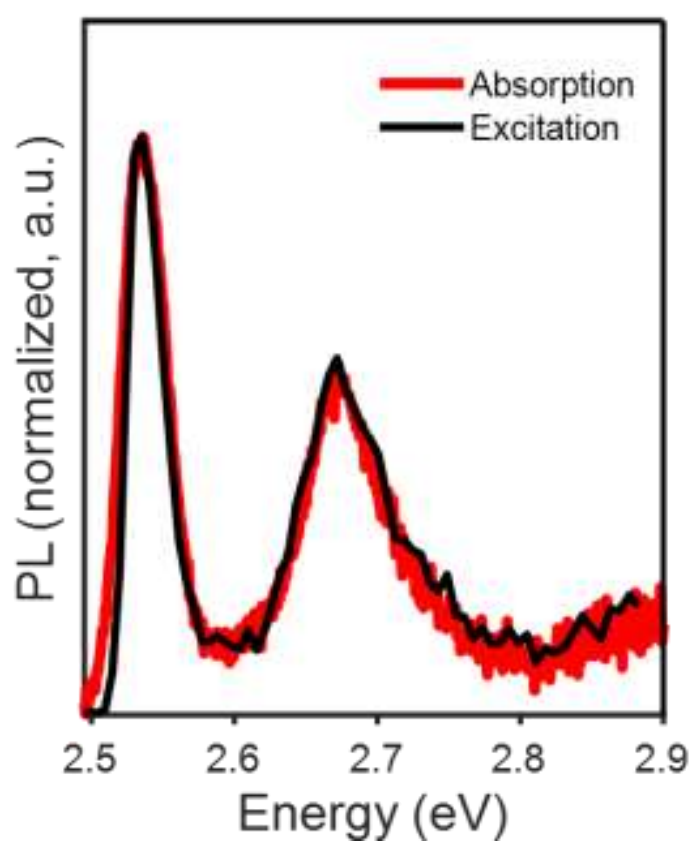

Figure S6. Excitation spectrum (black) of CdSe NPLs taken at 77K, with an emission wavelength of $520 \mathrm{~nm}$. The absorption spectrum (red) was taken at $77 \mathrm{~K}$ in a closed-cycle helium cryostat (see above for details).

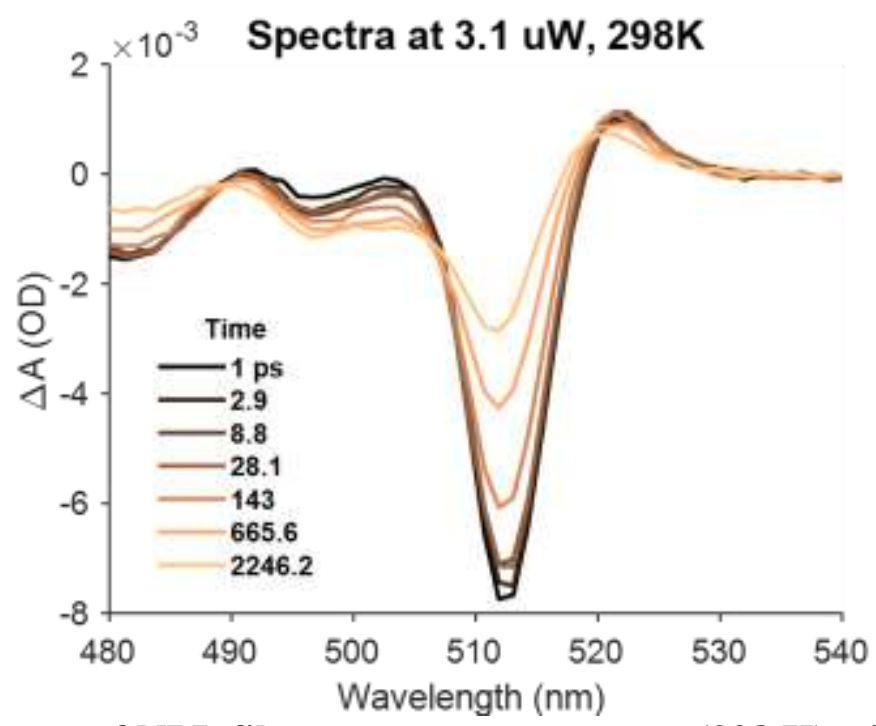

Figure S7. TA Spectrum of NPL film at room temperature $(298 \mathrm{~K})$ with a $3.1 \mathrm{uW}$ excitation power. The stimulated emission feature red-shifted from the exciton bleach we assign to trion emission only appears at low temperature and is not present at room temperature. 
Determination of $\langle\mathbf{N}\rangle$. The absorption cross section of the core-only $\sim 200 \mathrm{~nm}^{2} 4.5 \mathrm{ML} \mathrm{CdSe}$ NPLs measured at $85 \mathrm{~K}$ was calculated using experimental values from the literature (which was measured at $298 \mathrm{~K})^{8}$. In order to factor in temperature effects and our specific excitation energy, we performed two corrections. First, the increase in band-edge hh absorption when going from $298 \mathrm{~K}$ to $85 \mathrm{~K}$ was accounted for by comparing the relative increase in absorption of the hh bandedge feature on the TA film sample between the two temperatures. This resulted in an additional multiplicative correction factor $(\sim 1.8)$ for the hh absorption value derived for NPLs at $298 \mathrm{~K}$. Second, the absorption cross-section at our excitation wavelength of $2.63 \mathrm{eV}(470 \mathrm{~nm})$ is smaller than the hh band-edge absorption at $85 \mathrm{~K}$. Accounting for this absorption reduction gives a second correction factor of 0.26 . Combined, this resulted in an estimated absorption coefficient of $2.4 \times$ $10^{-14} \mathrm{~cm}^{2}$ at $85 \mathrm{~K}$.

Our laser repetition rate is $500 \mathrm{~Hz}$, and our excitation wavelength was $470 \mathrm{~nm}$. We measured our pump laser spot size by attaching a flat-edged blade perpendicular to the beam focus, located at the sample position. We measured the change in transmitted power as a function of blade translation position and obtained an error function, which can be fit to extract a Gaussian beam standard deviation $\sigma_{\mathrm{x}}$ of $40 \mu \mathrm{m}$ used for the spot size. We use a standard formula for $\langle\mathrm{N}\rangle$,

$$
\langle\mathrm{N}\rangle=\frac{C_{a b s} \times \text { power }}{\text { Photon energy } \times \text { Spot size } \times \text { Laser Repetition Rate }}
$$

For pump fluences used $(0.6 \mu \mathrm{W}-60 \mu \mathrm{W}),\langle\mathrm{N}\rangle=0.7$ - 68. Using a similar procedure, other authors have reported large values of $\langle\mathrm{N}\rangle$ at comparable excitation fluences. There is literature precedent that single NPLs can contain many excitons when optically excited, which can explain why our calculated $\langle\mathrm{N}\rangle$ seems high, when compared to quantum $\operatorname{dots}^{9,10}$.

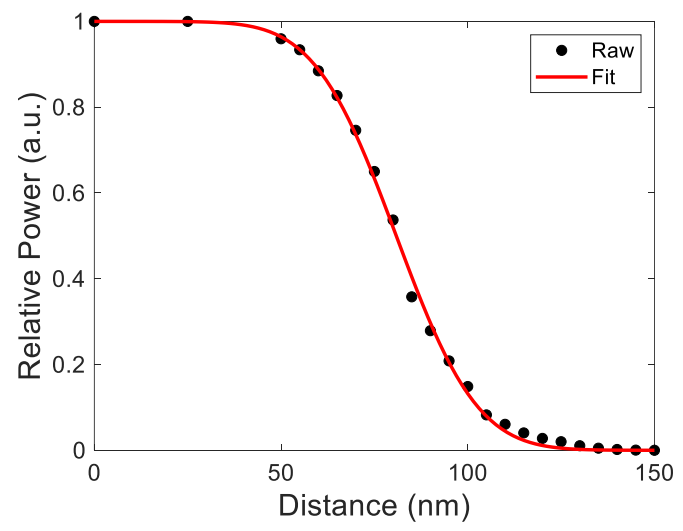

Figure S8. Beam intensity profile and fitted error function. 
Transient Absorption Fitting. As stated in the text, we fit all XB and $\mathrm{X}^{-} \mathrm{SE}$ kinetic traces with a multiexponential function: $-\Delta A=\sum-A_{i} \exp \left(t / \tau_{i}\right)$. The smallest exponential time constant was allowed to vary between 0.1 to $1 \mathrm{ps}$, to account for spectral shifting occurring between 0.3 and 1 ps within the IRF in addition to the IRF itself (described in the text). Additional time constants were allowed to vary from 0.6 ps to $10 \mathrm{~ns}$. The number of components was increased until the residual was scattered uniformly about 0 across the entire range of probe time delays. All raw data and corresponding fits can be found in Figure S10. A summary table of fitted amplitudes and constants can be found in Tables S1,2.

To clarify the interpretation of amplitudes, negative amplitudes are signals that either describe the rise of a negative signal, or describe a signal with positive $\Delta A$ signal (e.g. ESA). Positive amplitudes describe a negative $\Delta A$ signal (bleach or stimulated emission).

Fitting Robustness at Different Wavelengths. The wavelengths used for XB and $\mathrm{X}^{-} \mathrm{SE}$ in the main text were chosen to maximize signal amplitudes while minimizing spectral overlap (see Figure 2b, inset). We also fit the XB at 494 and $495 \mathrm{~nm}$, and the $\mathrm{X}^{-} \mathrm{SE}$ at 501 and $504 \mathrm{~nm}$. The tables for these fitted constants can be found in Tables S3-S6.

Assignment of Fitted TA Constants. We monitored the dynamics at wavelengths of $493 \mathrm{~nm}$ and $502 \mathrm{~nm}$ for the $\mathrm{XB}$ and $\mathrm{X}^{-} \mathrm{SE}$, respectively. We assign $\tau_{3}(\mathrm{XB})$ to bright exciton decay, based on comparison to time-resolved photoluminescence (TRPL) values of 10-50ps for NPLs of similar size $e^{5,11}$. Concerning $\tau_{4}(\mathrm{XB})$, we expect that biexciton and trion signals should scale similarly with power if trions form from biexcitons. Since we confidently assign $\tau_{4}\left(\mathrm{X}^{-} \mathrm{SE}\right)$ to trion radiative decay (this will be justified in the next paragraph), we designate $\tau_{4}(\mathrm{XB})$ as biexciton decay, since its amplitude scales similarly to $\tau_{4}\left(\mathrm{X}^{-} \mathrm{SE}\right)$ with respect to power (Figure S9c). Finally, we assign $\tau_{5}(\mathrm{XB})$ to decay of the dark exciton (bleach), supported by comparison to TRPL lifetime measurements of 10-30 $\mathrm{ns}^{11-13}$.

$\tau 3\left(\mathrm{X}^{-} \mathrm{SE}\right)$ has a non-negligible contribution to the rise of the trion SE signal. $\tau_{3}$ (i) is not IRFlimited, (ii) is not shared by the biexciton and exciton bleach and (iii) does not have an obvious trend with incident power. These observations rule out direct trion absorption and collisional formation of trions as the source of $\tau_{3}$. Due to a large spectral overlap between the exciton ESA and trion bleach, in addition to a large statistical uncertainty in the $\tau_{3}$ fit at some powers, we suspect 
that $\tau_{3}$ (in the $\mathrm{X}^{-\mathrm{SE}} \mathrm{E}$ dynamics) is a statistical mixture of the decay of the exciton ESA and the trion formation time constant $\tau_{2}$. (Figure $\left.\mathbf{S 9 b}\right)$. We assign $\tau_{4}\left(\mathrm{X}^{-} \mathrm{SE}\right)$ to trion radiative decay, due to similarities in magnitude to trion decay characterized by TRPL $(300-450 \mathrm{ps})^{5,12} \cdot \tau_{4}\left(\mathrm{X}^{-} \mathrm{SE}\right)$ is also the only positive amplitude component in $\mathrm{X}^{-} \mathrm{SE}$, which must describe the decay of an emitting species, the trion. We attribute $\tau 5\left(\mathrm{X}^{-} \mathrm{SE}\right)$ to the ESA of the dark exciton to a higher excited state manifold (see main text), since it shares a similarly long decay lifetime compared to $\tau_{5}(\mathrm{XB})$ but is opposite in amplitude.
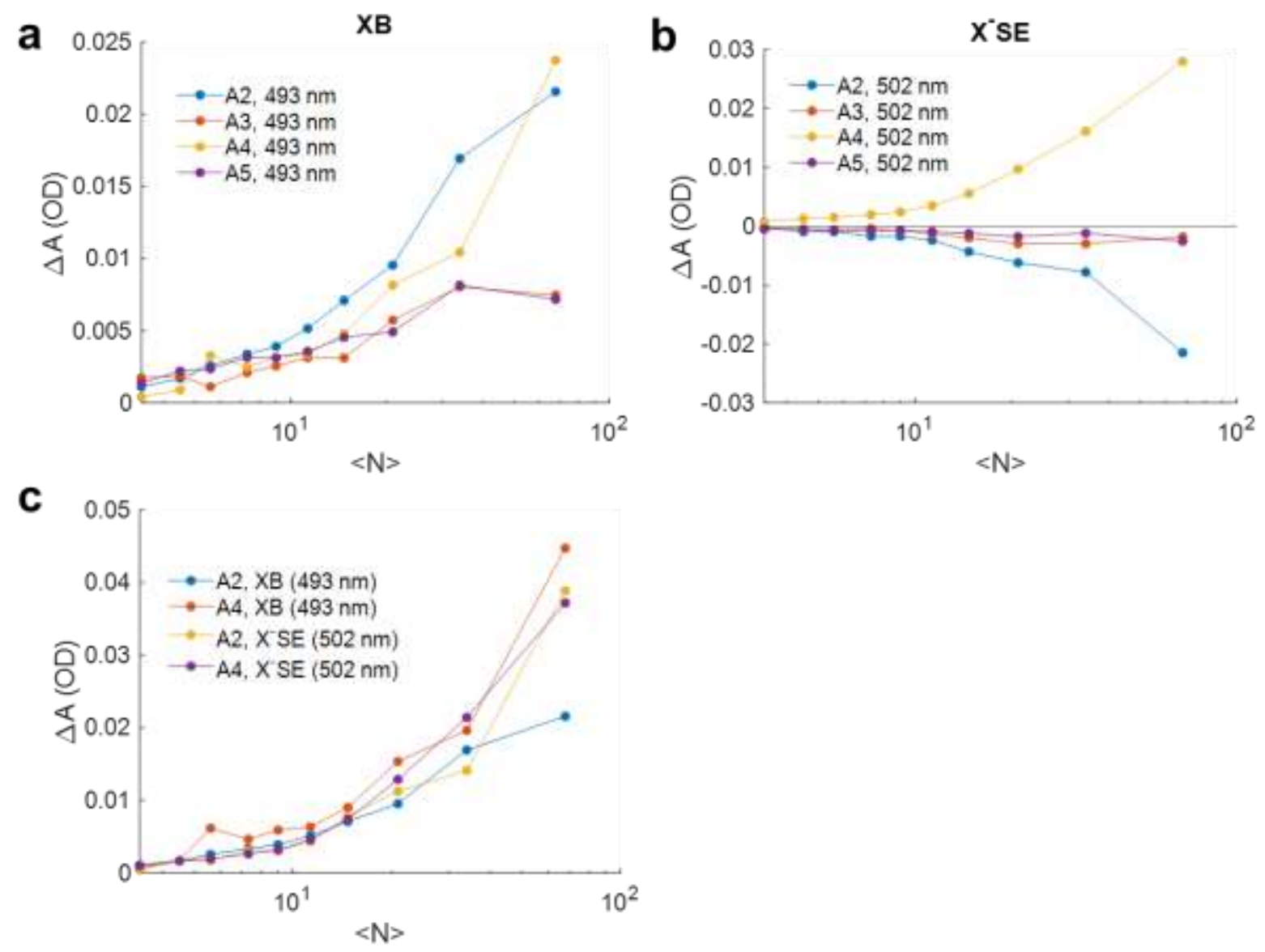

Fig S9. Amplitude scaling of fitted time constants $\tau_{2}$ to $\tau_{5}$ with respect to $\langle N\rangle$. (a) XB amplitudes. (b) $\mathrm{X}^{\top} \mathrm{SE}$ amplitudes. (c) Normalized A2/A4 showing similar scaling for $\mathrm{XB} / \mathrm{X}^{\top} \mathrm{SE}$ 

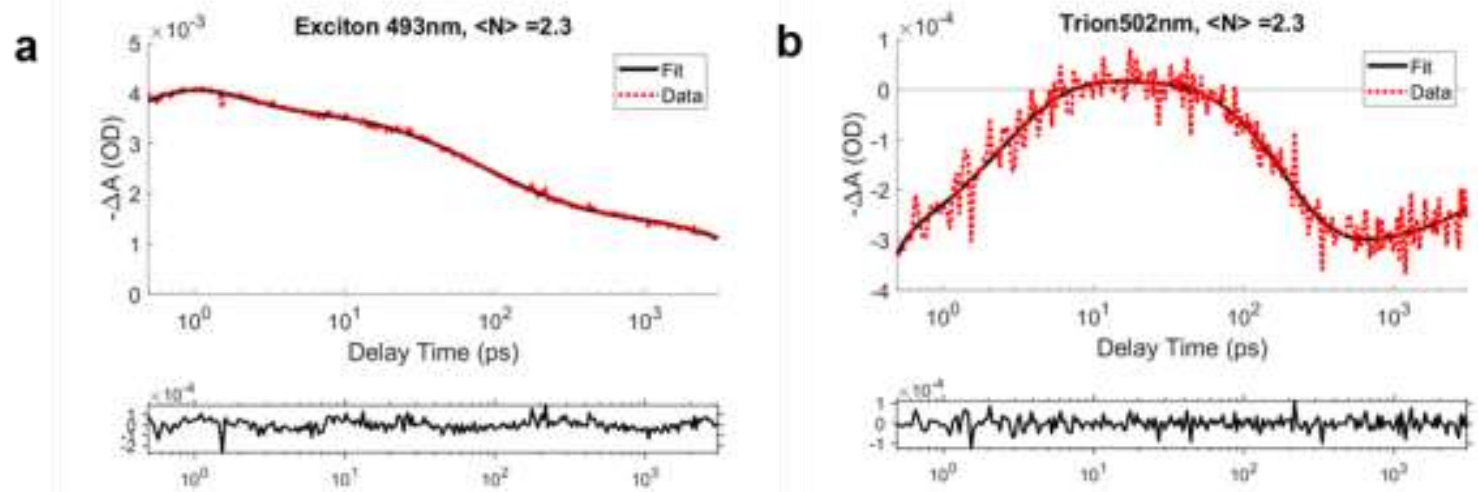

c
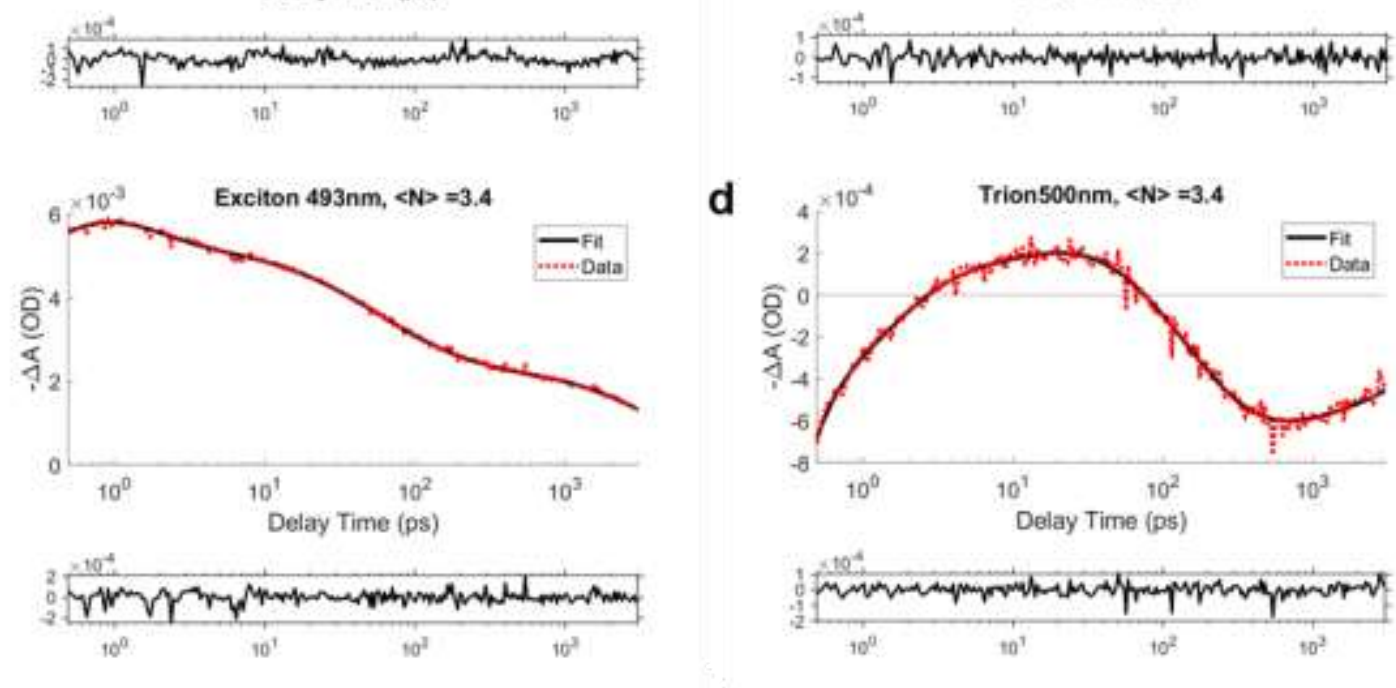

c
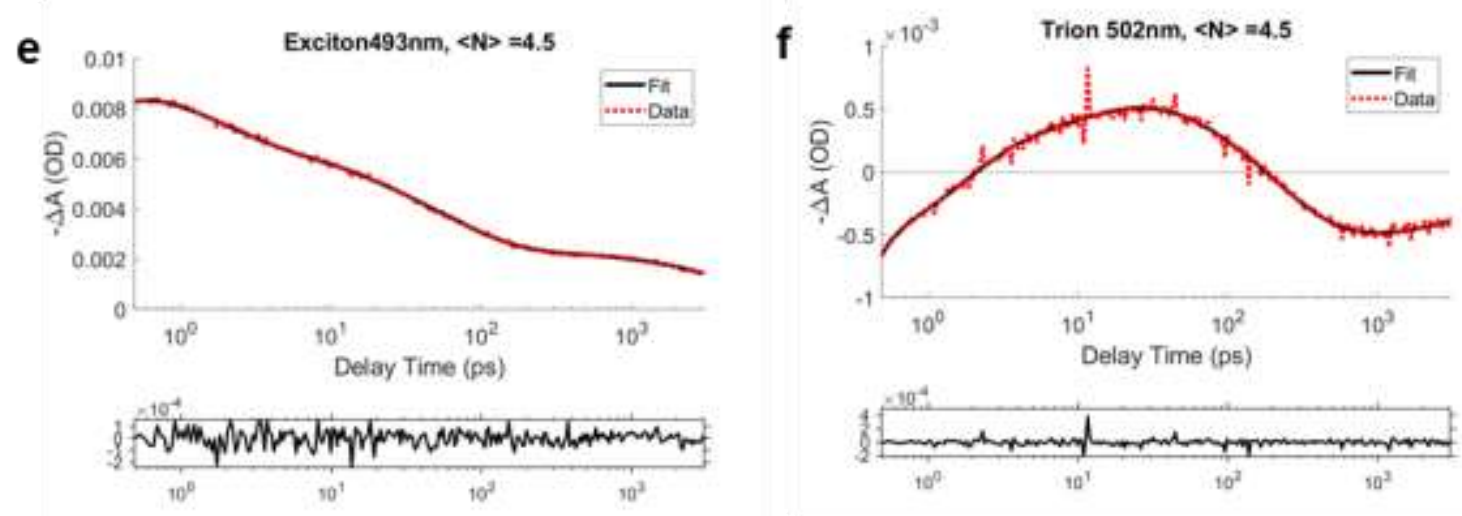
g
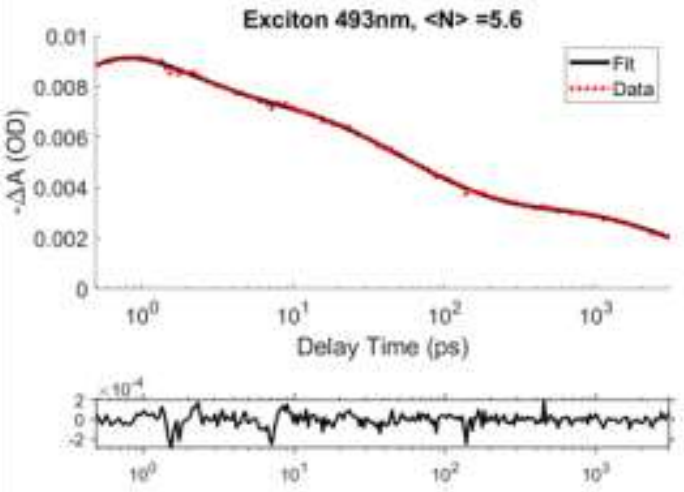

i
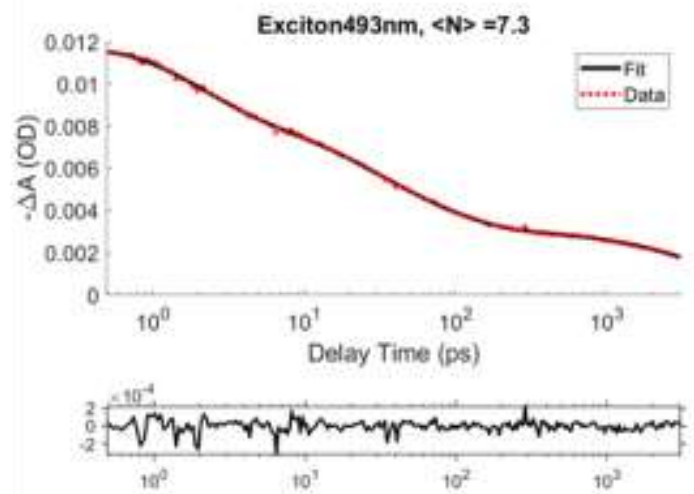

k

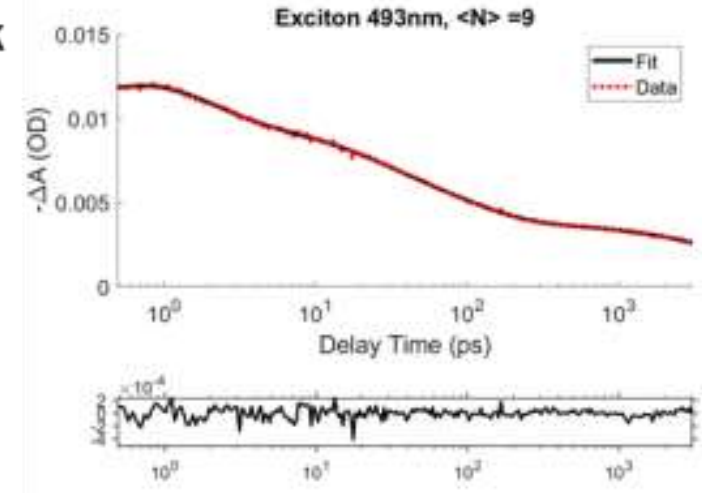

h
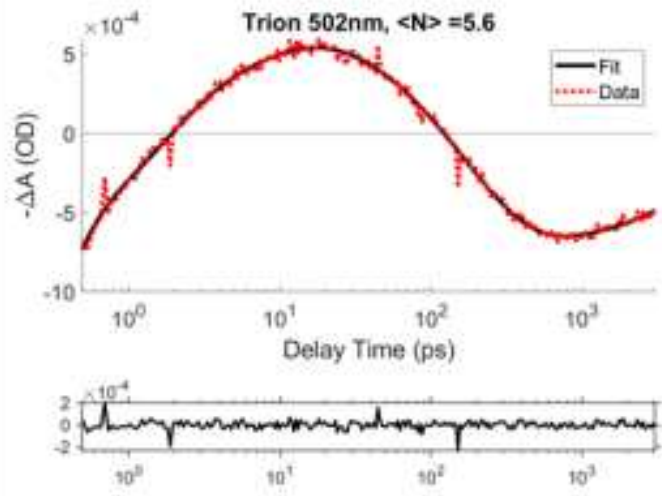

j
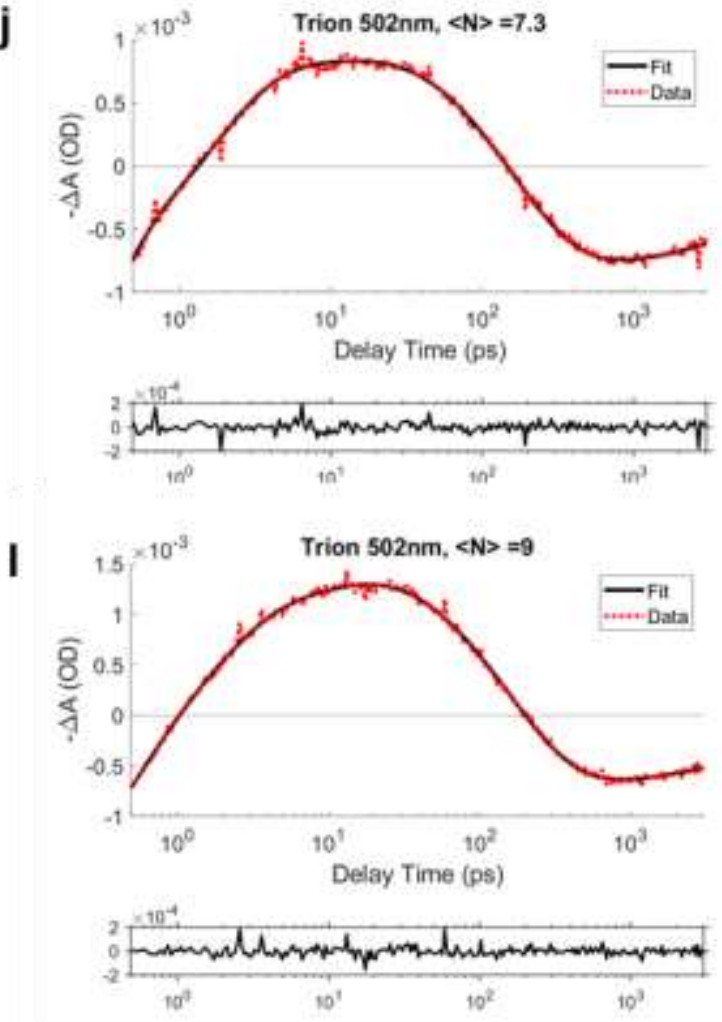
m
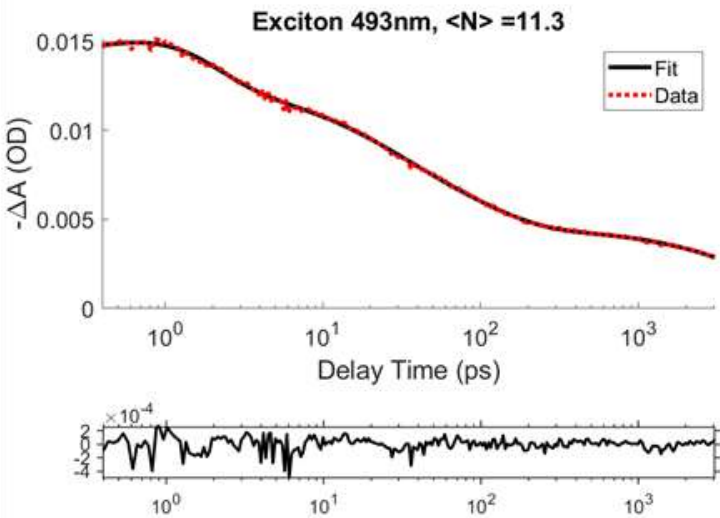

0
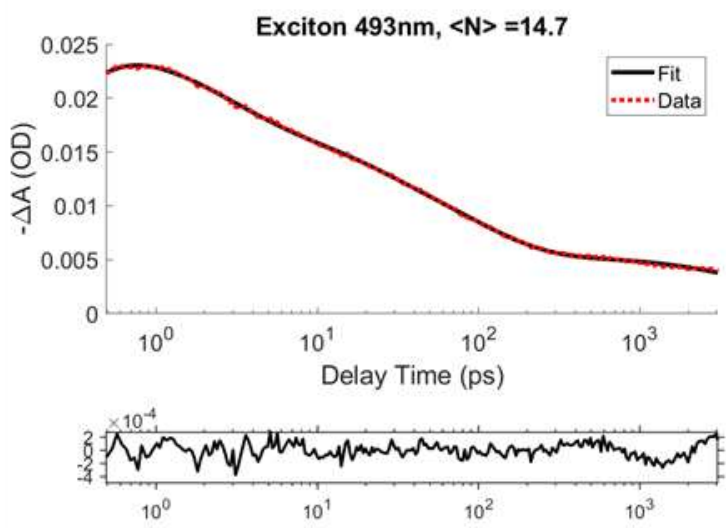

$q$

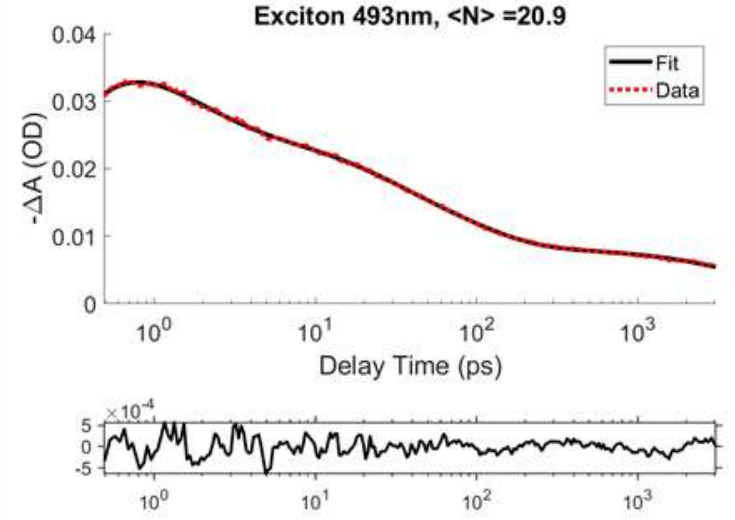

n
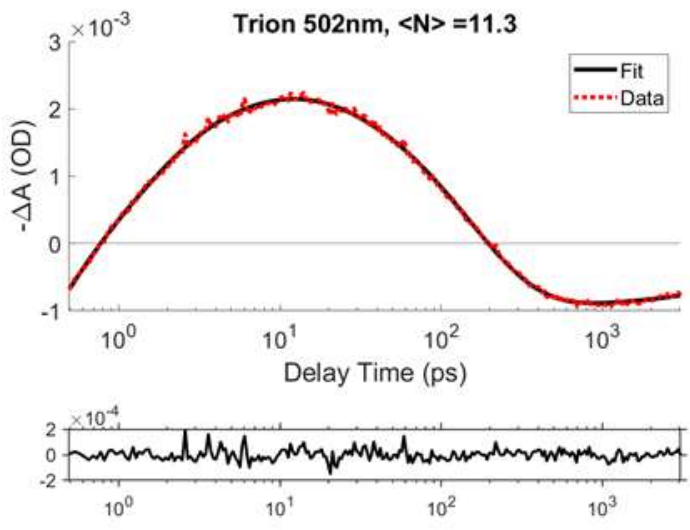

p
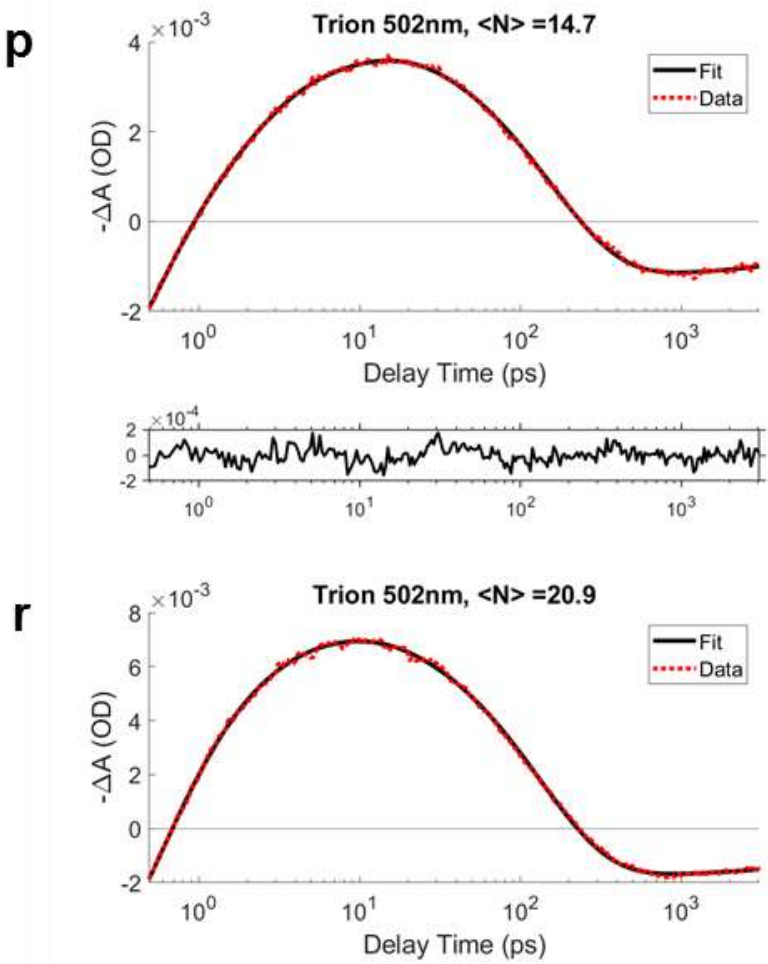

$\underset{10^{0}}{2} \underset{10^{1}}{2.10^{-4}}$ 


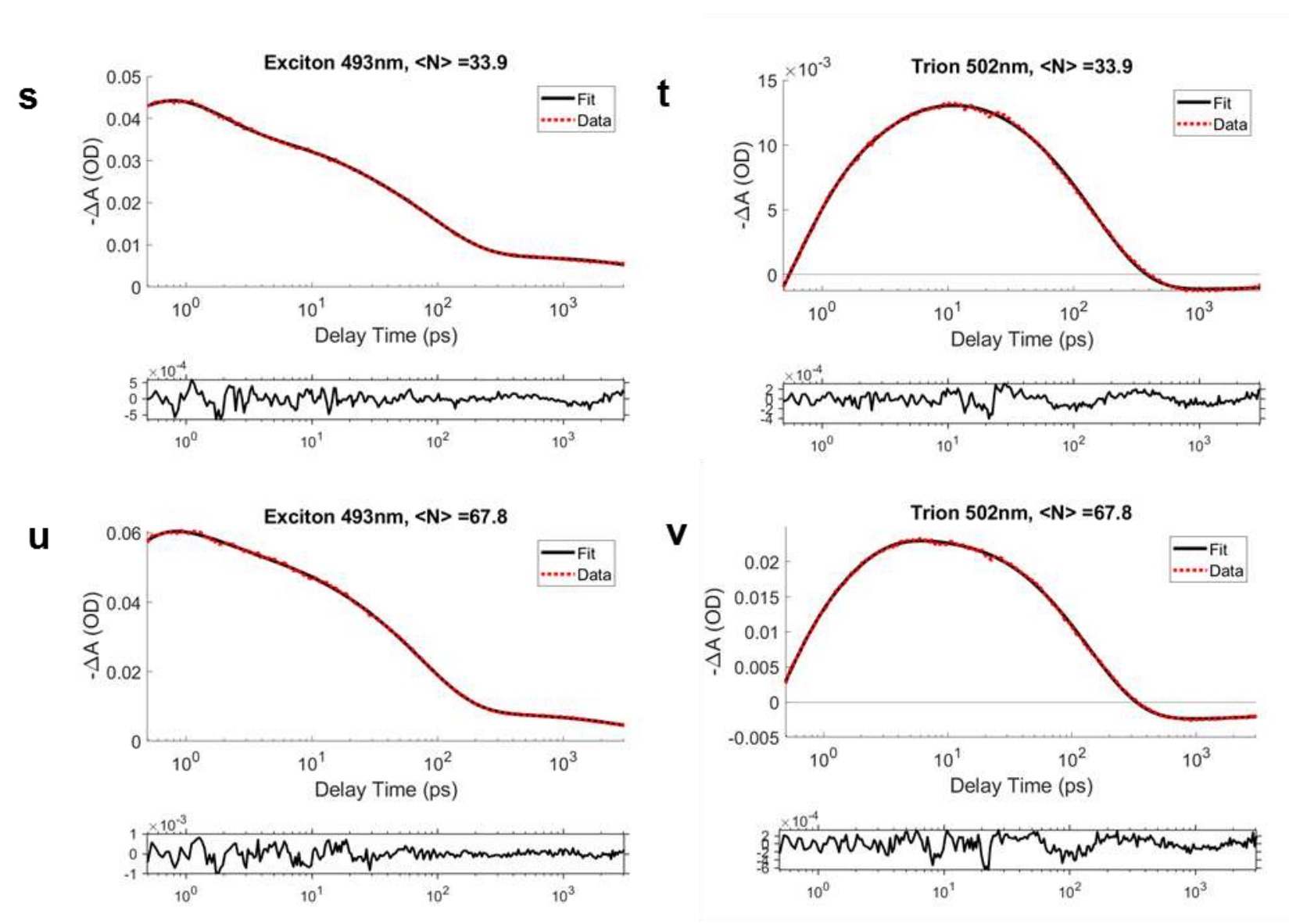

Figure S10. TA time trace (red), and fits (black) for 4 ML NPLs at $493 \mathrm{~nm}(\mathrm{XB})$ and $502 \mathrm{~nm}\left(\mathrm{X}^{-}\right.$ SE) for a range of pump fluences, taken at $85 \mathrm{~K}$. Residuals are plotted below the data. Plots are arranged in order of increasing pump fluence. Plot title describes the signal type and wavelength, as well as the pump fluence converted to $\langle\mathrm{N}\rangle$. (a) $\mathrm{XB}$ at $\langle\mathrm{N}\rangle=2.3$. (b) $\mathrm{X}^{-} \mathrm{SE}$ at $\langle\mathrm{N}\rangle=2.3$. (c) $\mathrm{XB}$ at $\langle\mathrm{N}\rangle=$ 3.4. (d) $\mathrm{X}^{-} \mathrm{SE}$ at $\langle\mathrm{N}\rangle=$ 3.4. (e) $\mathrm{XB}$ at $\langle\mathrm{N}\rangle=$ 4.5. (f) $\mathrm{X}^{-} \mathrm{SE}$ at $\langle\mathrm{N}\rangle=4.5$. (g) $\mathrm{XB}$ at $\langle\mathrm{N}\rangle=$ 5.6. (h) $\mathrm{X}^{-} \mathrm{SE}$ at $\langle\mathrm{N}\rangle=$ 5.6. (i) $\mathrm{XB}$ at $\langle\mathrm{N}\rangle=$ 7.3. (j) $\mathrm{X}^{-} \mathrm{SE}$ at $\langle\mathrm{N}\rangle=$ 7.3. (k) $\mathrm{XB}$ at $\langle\mathrm{N}\rangle=$ 9. (l) $\mathrm{X}^{-} \mathrm{SE}$ at $\langle\mathrm{N}\rangle$ = 9. (m) $X B$ at $\langle\mathrm{N}\rangle=$ 11.3. (n) $\mathrm{X}^{-} \mathrm{SE}$ at $\langle\mathrm{N}\rangle=11.3$. (o) $\mathrm{XB}$ at $\langle\mathrm{N}\rangle=14.7$. (p) $\mathrm{X}^{-} \mathrm{SE}$ at $\langle\mathrm{N}\rangle=14.7$. (q) $X B$ at $\langle N\rangle=20.9$. (r) $X^{-} S E$ at $\langle N\rangle=20.9$. (s) $X B$ at $\langle N\rangle=$ 33.9. (t) $X^{-} S E$ at $\langle N\rangle=$ 33.9. (u) $X B$ at $\langle\mathrm{N}\rangle=67.8$. (v) $\mathrm{X}^{-} \mathrm{SE}$ at $\langle\mathrm{N}\rangle=67.8$. 


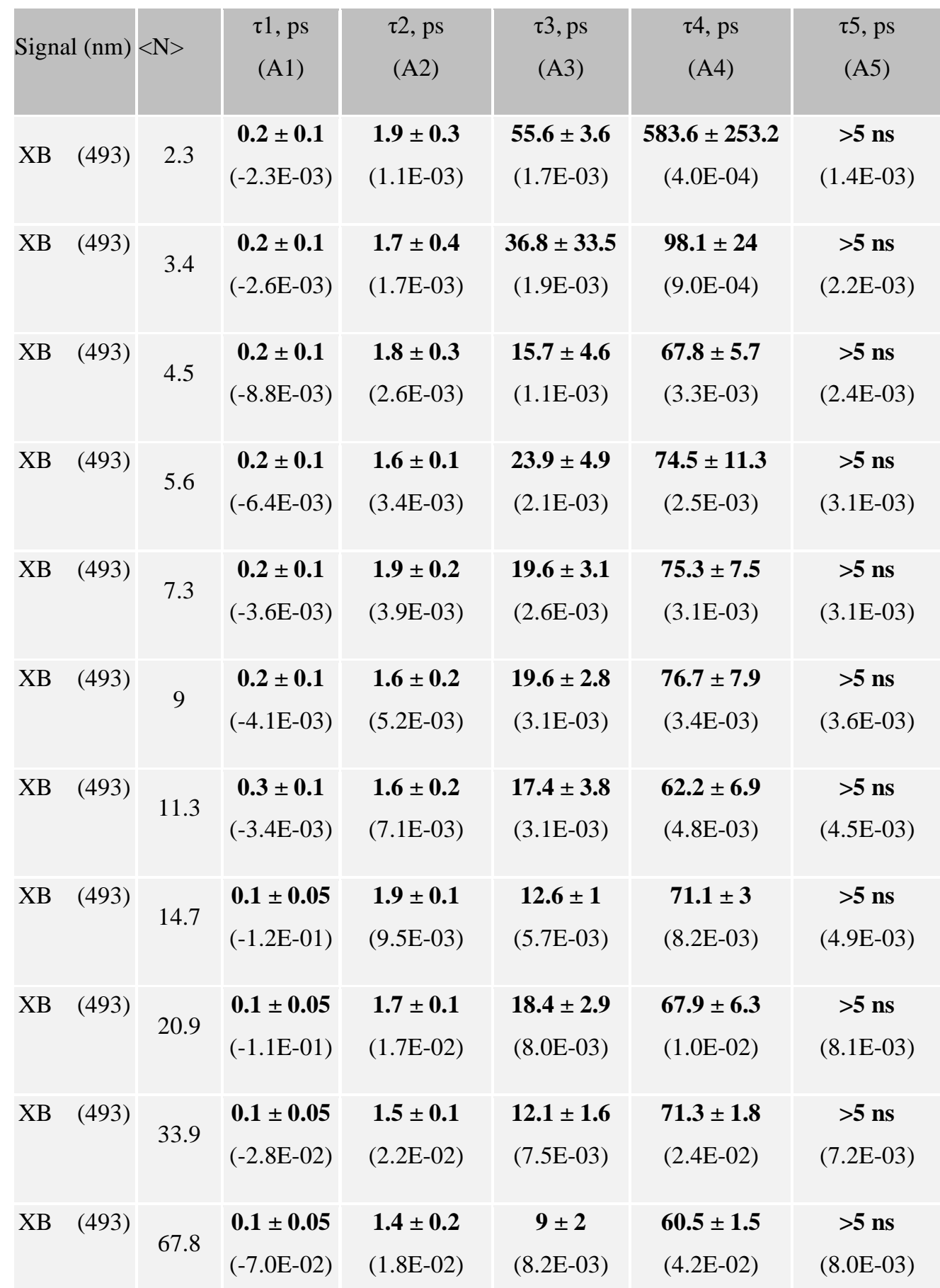

Table S1. Time constants and amplitudes of fit components for the dynamics of the exciton bleach $(\mathrm{XB}, 493 \mathrm{~nm}$ ) signal extracted from TA spectra of CdSe NPLs at $85 \mathrm{~K}$. Fitted time constants larger than $5 \mathrm{~ns}$ are not explicitly reported, due to the limited length of our optical track. 


\begin{tabular}{|c|c|c|c|c|c|c|}
\hline Signal (nm) & $<\mathrm{N}\rangle$ & $\begin{array}{l}\tau 1 \mathrm{ps} \\
\text { (A1) }\end{array}$ & $\begin{array}{l}\tau 2 \mathrm{ps} \\
\text { (A2) }\end{array}$ & $\begin{array}{l}\tau 3 \mathrm{ps} \\
\text { (A3) }\end{array}$ & $\begin{array}{l}\tau 4 \mathrm{ps} \\
\text { (A4) }\end{array}$ & $\begin{array}{l}\tau 5 \mathrm{ps} \\
\text { (A5) }\end{array}$ \\
\hline $\mathrm{X}^{-} \mathrm{SE}(502)$ & 2.3 & $\begin{array}{c}\mathbf{0 . 6} \pm \mathbf{0 . 2} \\
(-2.7 \mathrm{E}-04)\end{array}$ & $\begin{array}{c}\mathbf{4 . 5} \pm \mathbf{1 . 2} \\
(-3.05 \mathrm{E}-04)\end{array}$ & -- & $\begin{array}{c}\mathbf{2 0 5 . 5} \pm \mathbf{2 7} \\
(4.1 \mathrm{E}-04)\end{array}$ & $\begin{array}{c}>\mathbf{5} \mathbf{~ n s} \\
(-3.4 \mathrm{E}-04)\end{array}$ \\
\hline $\mathrm{X}^{-} \mathrm{SE}(502)$ & 3.4 & $\begin{array}{c}\mathbf{0 . 4} \pm \mathbf{0 . 2} \\
(-4.3 \mathrm{E}-04)\end{array}$ & $\begin{array}{c}2.3 \pm 1.1 \\
(-2.4 \mathrm{E}-04)\end{array}$ & $\begin{array}{r}\mathbf{1 3 . 4} \pm \mathbf{5 . 5} \\
(-3.1 \mathrm{E}-04)\end{array}$ & $\begin{array}{c}\mathbf{1 8 7 . 2} \pm \mathbf{2 2 . 7} \\
(7.5 \mathrm{E}-04)\end{array}$ & $\begin{array}{c}>\mathbf{5} \mathbf{~ n s} \\
(-5.0 \mathrm{E}-04)\end{array}$ \\
\hline $\mathrm{X}^{-\mathrm{SE}}$ & 4.5 & $\begin{array}{c}\mathbf{0 . 2} \pm \mathbf{0 . 1} \\
(-1.5 \mathrm{E}-02)\end{array}$ & $\begin{array}{c}1.5 \pm \mathbf{0 . 4} \\
(-9.4 \mathrm{E}-04)\end{array}$ & $\begin{array}{l}11.9 \pm 2.7 \\
(-5.7 \mathrm{E}-04)\end{array}$ & $\begin{array}{c}\mathbf{2 1 0 . 2} \pm \mathbf{1 7 . 4} \\
(1.3 \mathrm{E}-03)\end{array}$ & $\begin{array}{c}>\mathbf{5} \mathbf{~ n s} \\
(-5.5 \mathrm{E}-04)\end{array}$ \\
\hline $\mathrm{X}^{-\mathrm{SE}}$ & 5.6 & $\begin{array}{c}\mathbf{0 . 2} \pm \mathbf{0 . 1} \\
(-5.5 \mathrm{E}-03)\end{array}$ & $\begin{array}{c}\mathbf{1 . 4} \pm \mathbf{0 . 4} \\
(-9.6 \mathrm{E}-04)\end{array}$ & $\begin{array}{c}\mathbf{6 . 9} \pm \mathbf{1 . 5} \\
(-6.3 \mathrm{E}-04)\end{array}$ & $\begin{array}{c}\mathbf{1 7 3 . 6} \pm \mathbf{8 . 7} \\
(1.5 \mathrm{E}-03)\end{array}$ & $\begin{array}{c}>\mathbf{5} \mathbf{~ n s} \\
(-7.4 \mathrm{E}-04)\end{array}$ \\
\hline $\mathrm{X}^{-} \mathrm{SE}$ & 7.3 & $\begin{array}{c}\mathbf{0 . 2} \pm \mathbf{0 . 1} \\
(-5.6 \mathrm{E}-03)\end{array}$ & $\begin{array}{r}\mathbf{1 . 6} \pm \mathbf{0 . 2} \\
(-1.7 \mathrm{E}-03)\end{array}$ & $\begin{array}{l}\mathbf{1 5 . 3} \pm \mathbf{4 . 1} \\
(-4.2 \mathrm{E}-04)\end{array}$ & $\begin{array}{c}\mathbf{1 6 2 . 8} \pm \mathbf{8 . 4} \\
(2.0 \mathrm{E}-03)\end{array}$ & $\begin{array}{c}>\mathbf{5} \mathbf{~ n s} \\
(-8.2 \mathrm{E}-04)\end{array}$ \\
\hline $\mathrm{X}^{-} \mathrm{SE}$ & 9 & $\begin{array}{c}\mathbf{0 . 2} \pm \mathbf{0 . 1} \\
(-1.7 \mathrm{E}-03)\end{array}$ & $\begin{array}{c}\mathbf{1 . 5} \pm \mathbf{0 . 3} \\
(-1.7 \mathrm{E}-03)\end{array}$ & $\begin{array}{l}\mathbf{1 0 . 7} \pm \mathbf{1 . 9} \\
(-7.8 \mathrm{E}-04)\end{array}$ & $\begin{array}{c}\mathbf{1 6 1 . 9} \pm \mathbf{5 . 9} \\
(2.4 \mathrm{E}-03)\end{array}$ & $\begin{array}{c}>\mathbf{5} \mathbf{~ n s} \\
(-7.1 \mathrm{E}-04)\end{array}$ \\
\hline $\mathrm{X}-\mathrm{SE}$ & 11.3 & $\begin{array}{c}\mathbf{0 . 3} \pm \mathbf{0 . 1} \\
(-2.9 \mathrm{E}-03)\end{array}$ & $\begin{array}{c}\mathbf{1 . 2} \pm \mathbf{0 . 3} \\
(-2.4 \mathrm{E}-03)\end{array}$ & $\begin{array}{c}\mathbf{4 . 8} \pm \mathbf{0 . 9} \\
(-1.3 \mathrm{E}-03)\end{array}$ & $\begin{array}{c}\mathbf{1 5 0 . 3} \pm \mathbf{3 . 4} \\
(3.5 \mathrm{E}-03)\end{array}$ & $\begin{array}{c}>\mathbf{5} \mathbf{~ n s} \\
(-9.5 \mathrm{E}-04)\end{array}$ \\
\hline $\mathrm{X}-\mathrm{SE}$ & 14.7 & $\begin{array}{c}\mathbf{0 . 3} \pm \mathbf{0 . 1} \\
(-5.6 \mathrm{E}-03)\end{array}$ & $\begin{array}{c}\mathbf{1 . 4} \pm \mathbf{0 . 3} \\
(-4.3 \mathrm{E}-03)\end{array}$ & $\begin{array}{c}\mathbf{6 . 9} \pm \mathbf{6 . 9} \\
(-2.0 \mathrm{E}-03)\end{array}$ & $\begin{array}{c}\mathbf{1 5 5 . 4} \pm \mathbf{1 . 5} \\
(5.5 \mathrm{E}-03)\end{array}$ & $\begin{array}{c}>\mathbf{5} \mathbf{~ n s} \\
(-1.2 \mathrm{E}-03)\end{array}$ \\
\hline $\mathrm{X}^{-\mathrm{SE}}$ & 20.9 & $\begin{array}{c}\mathbf{0 . 3} \pm \mathbf{0 . 1} \\
(-1.1 \mathrm{E}-02)\end{array}$ & $\begin{array}{c}\mathbf{1 . 2} \pm \mathbf{0 . 5} \\
(-6.2 \mathrm{E}-03)\end{array}$ & $\begin{array}{c}4.2 \pm 1 \\
(-3.0 \mathrm{E}-03)\end{array}$ & $\begin{array}{c}\mathbf{1 3 2 . 5} \pm \mathbf{2 . 1} \\
(9.7 \mathrm{E}-03)\end{array}$ & $\begin{array}{c}>\mathbf{5} \mathbf{~ n s} \\
(-1.8 \mathrm{E}-03)\end{array}$ \\
\hline$X^{-S E}(502)$ & 33.9 & $\begin{array}{c}\mathbf{0 . 4} \pm \mathbf{0 . 1} \\
(-1.8 \mathrm{E}-02)\end{array}$ & $\begin{array}{c}\mathbf{1 . 9} \pm \mathbf{0 . 5} \\
(-7.8 \mathrm{E}-03)\end{array}$ & $\begin{array}{c}7.2 \pm \mathbf{2} \\
(-3.0 \mathrm{E}-03)\end{array}$ & $\begin{array}{c}\mathbf{1 4 5 . 9} \pm \mathbf{2 . 3} \\
(1.6 \mathrm{E}-02)\end{array}$ & $\begin{array}{c}>\mathbf{5} \mathbf{~ n s} \\
(-1.2 \mathrm{E}-03)\end{array}$ \\
\hline $\mathrm{X}^{-} \mathrm{SE}(502)$ & 67.8 & $\begin{array}{c}\mathbf{0 . 3} \pm \mathbf{0 . 1} \\
(-3.5 \mathrm{E}-02)\end{array}$ & $\begin{array}{c}\mathbf{1 . 2} \pm \mathbf{0 . 2} \\
(-2.1 \mathrm{E}-02)\end{array}$ & $\begin{array}{l}\mathbf{1 1 . 5} \pm \mathbf{3 . 3} \\
(-1.8 \mathrm{E}-03)\end{array}$ & $\begin{array}{c}\mathbf{1 3 6 . 5} \pm \mathbf{2 . 1} \\
(2.8 \mathrm{E}-02)\end{array}$ & $\begin{array}{c}>\mathbf{5} \mathbf{~ n s} \\
(-2.6 \mathrm{E}-03)\end{array}$ \\
\hline
\end{tabular}

Table S2. Time constants and amplitudes of fit components for the dynamics of the exciton bleach $\left(\mathrm{X}^{-} \mathrm{SE}, 502 \mathrm{~nm}\right.$ ) signal extracted from TA spectra of CdSe NPLs at $85 \mathrm{~K}$. Fitted time constants larger than $5 \mathrm{~ns}$ are not explicitly reported, due to the limited length of our optical track. 


\begin{tabular}{|c|c|c|c|c|c|c|}
\hline Signal (nm) & $\langle\mathrm{N}\rangle$ & $\begin{array}{l}\tau 1 \mathrm{ps} \\
\text { (A1) }\end{array}$ & $\begin{array}{l}\tau 2 \mathrm{ps} \\
(\mathrm{A} 2)\end{array}$ & $\begin{array}{l}\tau 3 \mathrm{ps} \\
\text { (A3) }\end{array}$ & $\begin{array}{l}\tau 4 \mathrm{ps} \\
\text { (A4) }\end{array}$ & $\begin{array}{l}\tau 5 \mathrm{ps} \\
\text { (A5) }\end{array}$ \\
\hline XB (494) & 2.3 & $\begin{array}{c}\mathbf{0 . 4} \pm \mathbf{0 . 2} \\
(-2.2 \mathrm{E}-03)\end{array}$ & $\begin{array}{c}\mathbf{1 . 4} \pm \mathbf{0 . 4} \\
(1.0 \mathrm{E}-03)\end{array}$ & $\begin{array}{c}\mathbf{2 5 . 7} \pm \mathbf{1 4 . 6} \\
(4.4 \mathrm{E}-04)\end{array}$ & $\begin{array}{c}\mathbf{1 2 5} \pm \mathbf{2 7 . 9} \\
(1.6 \mathrm{E}-03)\end{array}$ & $\begin{array}{c}>\mathbf{5 n s} \\
(1.7 \mathrm{E}-03)\end{array}$ \\
\hline XB (494) & 3.4 & $\begin{array}{c}\mathbf{0 . 3} \pm \mathbf{0 . 1} \\
(-3.1 \mathrm{E}-03)\end{array}$ & $\begin{array}{r}\mathbf{1 . 4} \pm \mathbf{0 . 2} \\
(1.4 \mathrm{E}-03)\end{array}$ & $\begin{array}{c}\mathbf{2 9 . 3} \pm \mathbf{1 0 . 7} \\
(1.0 \mathrm{E}-03)\end{array}$ & $\begin{array}{c}\mathbf{9 8 . 2} \pm \mathbf{2 3 . 4} \\
(1.9 \mathrm{E}-03)\end{array}$ & $\begin{array}{c}>\mathbf{5 n s} \\
(2.4 \mathrm{E}-03)\end{array}$ \\
\hline XB (494) & 4.5 & $\begin{array}{c}\mathbf{0 . 2} \pm \mathbf{0 . 1} \\
(-6.1 \mathrm{E}-03)\end{array}$ & $\begin{array}{c}\mathbf{2 . 1} \pm \mathbf{0 . 4} \\
(1.8 \mathrm{E}-03)\end{array}$ & $\begin{array}{l}\mathbf{1 9 . 8} \pm \mathbf{5 . 4} \\
(9.7 \mathrm{E}-04)\end{array}$ & $\begin{array}{l}\mathbf{1 0 0 . 3} \pm \mathbf{7} \\
(3.3 \mathrm{E}-03)\end{array}$ & $\begin{array}{c}>\mathbf{5 n s} \\
(2.6 \mathrm{E}-03)\end{array}$ \\
\hline XB (494) & 5.6 & $\begin{array}{r}\mathbf{0 . 2} \pm \mathbf{0 . 2} \\
(-6.9 \mathrm{E}-03)\end{array}$ & $\begin{array}{l}\mathbf{1 . 9} \pm \mathbf{0 . 3} \\
(2.3 \mathrm{E}-03)\end{array}$ & $\begin{array}{c}\mathbf{2 5 . 9} \pm \mathbf{6 . 3} \\
(1.6 \mathrm{E}-03)\end{array}$ & $\begin{array}{l}\mathbf{8 9 . 2} \pm \mathbf{2 0} \\
(2.9 \mathrm{E}-03)\end{array}$ & $\begin{array}{c}>\mathbf{5 n s} \\
(3.4 \mathrm{E}-03)\end{array}$ \\
\hline XB (494) & 7.3 & $\begin{array}{c}\mathbf{0 . 2} \pm \mathbf{0 . 1} \\
(-5.7 \mathrm{E}-03)\end{array}$ & $\begin{array}{c}\mathbf{2 . 4} \pm \mathbf{0 . 4} \\
(2.5 \mathrm{E}-03)\end{array}$ & $\begin{array}{c}16 \pm \mathbf{2 . 8} \\
(2.2 \mathrm{E}-03)\end{array}$ & $\begin{array}{c}94.3 \pm 6 \\
(3.9 \mathrm{E}-03)\end{array}$ & $\begin{array}{c}>\mathbf{5 n s} \\
(3.1 \mathrm{E}-03)\end{array}$ \\
\hline XB (494) & 9 & $\begin{array}{c}\mathbf{0 . 3} \pm \mathbf{0 . 1} \\
(-5.0 \mathrm{E}-03)\end{array}$ & $\begin{array}{c}\mathbf{1 . 6} \pm \mathbf{0 . 2} \\
(4.1 \mathrm{E}-03)\end{array}$ & $\begin{array}{l}\mathbf{2 1 . 4} \pm \mathbf{3 . 5} \\
(2.5 \mathrm{E}-03)\end{array}$ & $\begin{array}{c}\mathbf{9 7} \pm \mathbf{8 . 4} \\
(3.8 \mathrm{E}-03)\end{array}$ & $\begin{array}{c}>\mathbf{5 n s} \\
(3.8 \mathrm{E}-03)\end{array}$ \\
\hline XB (494) & 11.3 & $\begin{array}{c}\mathbf{0 . 4} \pm \mathbf{0 . 1} \\
(-6.2 \mathrm{E}-03)\end{array}$ & $\begin{array}{r}\mathbf{1 . 5} \pm \mathbf{0 . 3} \\
(5.8 \mathrm{E}-03)\end{array}$ & $\begin{array}{c}\mathbf{2 0 . 2} \pm \mathbf{3 . 3} \\
(3.2 \mathrm{E}-03)\end{array}$ & $\begin{array}{c}\mathbf{8 8 . 5} \pm \mathbf{8} \\
(4.8 \mathrm{E}-03)\end{array}$ & $\begin{array}{c}>\mathbf{5 n s} \\
(4.5 \mathrm{E}-03)\end{array}$ \\
\hline XB (494) & 14.7 & $\begin{array}{c}\mathbf{0 . 2} \pm \mathbf{0 . 1} \\
(-1.8 \mathrm{E}-02)\end{array}$ & $\begin{array}{c}\mathbf{2 . 5} \pm \mathbf{0 . 3} \\
(7.4 \mathrm{E}-03)\end{array}$ & $\begin{array}{c}\mathbf{1 7 . 8} \pm \mathbf{2 . 6} \\
(4.3 \mathrm{E}-03)\end{array}$ & $\begin{array}{c}\mathbf{9 7 . 5} \pm \mathbf{4 . 8} \\
(8.7 \mathrm{E}-03)\end{array}$ & $\begin{array}{c}>\mathbf{5 n s} \\
(5.4 \mathrm{E}-03)\end{array}$ \\
\hline XB (494) & 20.9 & $\begin{array}{c}\mathbf{0 . 2} \pm \mathbf{0 . 1} \\
(-4.5 \mathrm{E}-02)\end{array}$ & $\begin{array}{c}\mathbf{1 . 7} \pm \mathbf{0 . 1} \\
(1.2 \mathrm{E}-02)\end{array}$ & $\begin{array}{c}\mathbf{2 1 . 5} \pm \mathbf{3 . 3} \\
(6.9 \mathrm{E}-03)\end{array}$ & $\begin{array}{c}\mathbf{8 7 . 4} \pm \mathbf{6 . 8} \\
(1.1 \mathrm{E}-02)\end{array}$ & $\begin{array}{c}>\mathbf{5 n s} \\
(8.4 \mathrm{E}-03)\end{array}$ \\
\hline XB (494) & 33.9 & $\begin{array}{c}\mathbf{0 . 3} \pm \mathbf{0 . 1} \\
(-3.0 \mathrm{E}-02)\end{array}$ & $\begin{array}{l}\mathbf{1 . 5} \pm \mathbf{0 . 2} \\
(1.6 \mathrm{E}-02)\end{array}$ & $\begin{array}{l}\mathbf{1 3 . 4} \pm \mathbf{1 . 9} \\
(5.5 \mathrm{E}-03)\end{array}$ & $\begin{array}{c}\mathbf{9 0 . 4} \pm \mathbf{2} \\
(2.4 \mathrm{E}-02)\end{array}$ & $\begin{array}{c}>\mathbf{5 n s} \\
(7.6 \mathrm{E}-03)\end{array}$ \\
\hline XB (494) & 67.8 & $\begin{array}{c}\mathbf{0 . 2} \pm \mathbf{0 . 1} \\
(-6.1 \mathrm{E}-02)\end{array}$ & $\begin{array}{c}\mathbf{1 . 5} \pm \mathbf{0 . 3} \\
(9.3 \mathrm{E}-03)\end{array}$ & $\begin{array}{c}9.6 \pm 2 \\
(6.9 \mathrm{E}-03)\end{array}$ & $\begin{array}{l}\mathbf{7 4 . 6} \pm \mathbf{1 . 5} \\
(4.2 \mathrm{E}-02)\end{array}$ & $\begin{array}{c}>\mathbf{5 n s} \\
(8.3 \mathrm{E}-03)\end{array}$ \\
\hline
\end{tabular}

Table S3. Time constants and amplitudes of fit components for the dynamics of the exciton bleach $(\mathrm{XB}, 494 \mathrm{~nm}$ ) signal extracted from TA spectra of CdSe NPLs at $85 \mathrm{~K}$. Fitted time constants larger than $5 \mathrm{~ns}$ are not explicitly reported, due to the limited length of our optical track. 


\begin{tabular}{|c|c|c|c|c|c|c|c|}
\hline \multicolumn{2}{|c|}{ Signal (nm) } & $\langle\mathrm{N}\rangle$ & $\begin{array}{l}\tau 1 \mathrm{ps} \\
(\mathrm{A} 1)\end{array}$ & $\begin{array}{c}\tau 2 \mathrm{ps} \\
(\mathrm{A} 2)\end{array}$ & $\begin{array}{c}\tau 3 \mathrm{ps} \\
(\mathrm{A} 3)\end{array}$ & $\begin{array}{c}\tau 4 \mathrm{ps} \\
(\mathrm{A} 4)\end{array}$ & $\begin{array}{l}\tau 5 \mathrm{ps} \\
(\mathrm{A} 5)\end{array}$ \\
\hline XB & (495) & 2.3 & $\begin{array}{c}0.2 \pm 0.1 \\
(-4.1 \mathrm{E}-03)\end{array}$ & $\begin{array}{c}3.8 \pm \mathbf{3} \\
(4.8 \mathrm{E}-04)\end{array}$ & $\begin{array}{c}7 \pm 6.2 \\
(-2.2 \mathrm{E}-04)\end{array}$ & $\begin{array}{c}132.2 \pm 9.7 \\
(1.5 \mathrm{E}-03)\end{array}$ & $\begin{array}{c}>\mathbf{5 n s} \\
(1.5 \mathrm{E}-03)\end{array}$ \\
\hline XB & (495) & 3.4 & $\begin{array}{c}0.3 \pm 0.1 \\
(-4.7 E-03)\end{array}$ & $\begin{array}{l}1.4 \pm 0.5 \\
(6.6 E-04)\end{array}$ & $\begin{array}{c}\mathbf{4 4 . 2} \pm \mathbf{1 4 . 3} \\
(4.6 \mathrm{E}-04)\end{array}$ & $\begin{array}{c}\mathbf{1 2 0 . 8} \pm \mathbf{2 9 . 4} \\
(1.7 \mathrm{E}-03)\end{array}$ & $\begin{array}{c}>\mathbf{5 n s} \\
(2.1 \mathrm{E}-03)\end{array}$ \\
\hline XB & (495) & 4.5 & $\begin{array}{c}0.3 \pm 0.1 \\
(-5.6 \mathrm{E}-03)\end{array}$ & $\begin{array}{l}\mathbf{2 . 6} \pm \mathbf{0 . 5} \\
(1.1 \mathrm{E}-03)\end{array}$ & $\begin{array}{c}\mathbf{4 9 . 4} \pm \mathbf{2 3 . 8} \\
(3.9 \mathrm{E}-04)\end{array}$ & $\begin{array}{c}132.5 \pm 27.6 \\
(2.9 \mathrm{E}-03)\end{array}$ & $\begin{array}{c}>\mathbf{5 n s} \\
(2.4 \mathrm{E}-03)\end{array}$ \\
\hline XB & (495) & 5.6 & $\begin{array}{c}\mathbf{0 . 3} \pm \mathbf{0 . 2} \\
(-5.6 \mathrm{E}-03)\end{array}$ & $\begin{array}{l}\mathbf{1 . 8} \pm \mathbf{0 . 4} \\
(1.5 \mathrm{E}-03)\end{array}$ & $\begin{array}{l}\mathbf{3 1 . 8} \pm 9.6 \\
(9.5 \mathrm{E}-04)\end{array}$ & $\begin{array}{c}116.4 \pm 14.3 \\
(2.6 \mathrm{E}-03)\end{array}$ & $\begin{array}{c}>\mathbf{5 n s} \\
(2.8 \mathrm{E}-03)\end{array}$ \\
\hline XB & (495) & 7.3 & $\begin{array}{c}0.3 \pm 0.1 \\
(-5.6 \mathrm{E}-03)\end{array}$ & $\begin{array}{l}\mathbf{2 . 5} \pm \mathbf{0 . 5} \\
(1.4 \mathrm{E}-03)\end{array}$ & $\begin{array}{c}\mathbf{2 0} \pm \mathbf{3 . 9} \\
(1.3 \mathrm{E}-03)\end{array}$ & $\begin{array}{c}120.6 \pm 6.7 \\
(3.5 \mathrm{E}-03)\end{array}$ & $\begin{array}{c}>\mathbf{5 n s} \\
(2.7 \mathrm{E}-03)\end{array}$ \\
\hline XB & (495) & 9 & $\begin{array}{c}\mathbf{0 . 4} \pm \mathbf{0 . 1} \\
(-5.8 \mathrm{E}-03)\end{array}$ & $\begin{array}{l}\mathbf{1 . 5} \pm \mathbf{0 . 3} \\
(2.5 \mathrm{E}-03)\end{array}$ & $\begin{array}{l}25.4 \pm 4.7 \\
(1.6 \mathrm{E}-03)\end{array}$ & $\begin{array}{c}116.2 \pm 9.4 \\
(3.6 \mathrm{E}-03)\end{array}$ & $\begin{array}{c}>\mathbf{5 n s} \\
(3.1 \mathrm{E}-03)\end{array}$ \\
\hline XB & (495) & 11.3 & $\begin{array}{c}\mathbf{0 . 4} \pm \mathbf{0 . 1} \\
(-7.3 \mathrm{E}-03)\end{array}$ & $\begin{array}{l}\mathbf{1 . 3} \pm \mathbf{0 . 3} \\
(3.8 \mathrm{E}-03)\end{array}$ & $\begin{array}{l}21.3 \pm 3.9 \\
(2.3 E-03)\end{array}$ & $\begin{array}{c}\mathbf{1 0 3 . 5} \pm \mathbf{8 . 1} \\
(4.7 \mathrm{E}-03)\end{array}$ & $\begin{array}{c}>\mathbf{5 n s} \\
(3.5 \mathrm{E}-03)\end{array}$ \\
\hline XB & (495) & 14.7 & $\begin{array}{c}\mathbf{0 . 3} \pm \mathbf{0 . 1} \\
(-1.8 \mathrm{E}-02)\end{array}$ & $\begin{array}{c}\mathbf{4} \pm \mathbf{0 . 3} \\
(4.6 \mathrm{E}-03)\end{array}$ & $\begin{array}{c}\mathbf{5 0} \pm \mathbf{7 . 7} \\
(4.8 \mathrm{E}-03)\end{array}$ & $\begin{array}{c}\mathbf{1 6 0 . 8} \pm \mathbf{2 0 . 1} \\
(5.5 \mathrm{E}-03)\end{array}$ & $\begin{array}{c}>\mathbf{5 n s} \\
(4.2 \mathrm{E}-03)\end{array}$ \\
\hline XB & (495) & 20.9 & $\begin{array}{c}0.2 \pm 0.1 \\
(-3.5 E-02)\end{array}$ & $\begin{array}{c}2 \pm \mathbf{0 . 3} \\
(6.2 \mathrm{E}-03)\end{array}$ & $\begin{array}{l}26.5 \pm 4.9 \\
(4.7 E-03)\end{array}$ & $\begin{array}{c}\mathbf{1 0 3 . 4} \pm \mathbf{7 . 6} \\
(1.1 \mathrm{E}-02)\end{array}$ & $\begin{array}{c}>\mathbf{5 n s} \\
(6.6 \mathrm{E}-03)\end{array}$ \\
\hline XB & (495) & 33.9 & $\begin{array}{c}\mathbf{0 . 3} \pm \mathbf{0 . 1} \\
(-2.9 \mathrm{E}-02)\end{array}$ & $\begin{array}{l}\mathbf{1 . 5} \pm \mathbf{0 . 2} \\
(7.5 \mathrm{E}-03)\end{array}$ & $\begin{array}{c}16 \pm \mathbf{3} \\
(3.3 E-03)\end{array}$ & $\begin{array}{c}\mathbf{1 0 7 . 3} \pm \mathbf{2 . 3} \\
(2.3 \mathrm{E}-02)\end{array}$ & $\begin{array}{c}>\mathbf{5 n s} \\
(5.8 \mathrm{E}-03)\end{array}$ \\
\hline XB & (495) & 67.8 & $\begin{array}{r}0.2 \pm 0.1 \\
(-4.7 E-02)\end{array}$ & $\begin{array}{l}\mathbf{3 . 8} \pm \mathbf{3 . 8} \\
(8.7 \mathrm{E}-04)\end{array}$ & $\begin{array}{c}\mathbf{1 2 . 5} \pm \mathbf{1 2 . 5} \\
(4.1 \mathrm{E}-03)\end{array}$ & $\begin{array}{l}\mathbf{8 8 . 3} \pm \mathbf{4 . 3} \\
(3.9 \mathrm{E}-02)\end{array}$ & $\begin{array}{c}>\mathbf{5 n s} \\
(6.1 \mathrm{E}-03)\end{array}$ \\
\hline
\end{tabular}

Table S4. Time constants and amplitudes of fit components for the dynamics of the exciton bleach $(\mathrm{XB}, 495 \mathrm{~nm}$ ) signal extracted from TA spectra of CdSe NPLs at $85 \mathrm{~K}$. Fitted time constants larger than $5 \mathrm{~ns}$ are not explicitly reported, due to the limited length of our optical track. 


\begin{tabular}{|c|c|c|c|c|c|c|}
\hline Signal (nm) & $\langle\mathrm{N}\rangle$ & $\begin{array}{l}\tau 1 \mathrm{ps} \\
\text { (A1) }\end{array}$ & $\begin{array}{l}\tau 2 \mathrm{ps} \\
\text { (A2) }\end{array}$ & $\begin{array}{l}\tau 3 \mathrm{ps} \\
\text { (A3) }\end{array}$ & $\begin{array}{l}\tau 4 \mathrm{ps} \\
\text { (A4) }\end{array}$ & $\begin{array}{l}\tau 5 \mathrm{ps} \\
\text { (A5) }\end{array}$ \\
\hline $\mathrm{X}$-SE (501) & 2.3 & $\begin{array}{c}\mathbf{0 . 7} \pm \mathbf{0 . 1} \\
(-6.8 \mathrm{E}-04)\end{array}$ & $\begin{array}{c}10 \pm \mathbf{2 . 2} \\
(-2.8 \mathrm{E}-04)\end{array}$ & -- & $\begin{array}{c}\mathbf{1 7 0 . 9} \pm \mathbf{1 4 . 8} \\
(6.7 \mathrm{E}-04)\end{array}$ & $\begin{array}{c}>\mathbf{5 n s} \\
(-4.6 \mathrm{E}-04)\end{array}$ \\
\hline $\mathrm{X}^{-} \mathrm{SE}$ & 3.4 & $\begin{array}{c}\mathbf{0 . 4} \pm \mathbf{0 . 2} \\
(-7.8 \mathrm{E}-04)\end{array}$ & $\begin{array}{c}\mathbf{1 . 7} \pm \mathbf{0 . 7} \\
(-5.3 \mathrm{E}-04)\end{array}$ & $\begin{array}{l}\mathbf{1 4 . 5} \pm \mathbf{4 . 2} \\
(-3.6 \mathrm{E}-04)\end{array}$ & $\begin{array}{c}\mathbf{1 6 6 . 8} \pm \mathbf{1 4 . 6} \\
(9.7 \mathrm{E}-04)\end{array}$ & $\begin{array}{c}>\mathbf{5 n s} \\
(-7.0 \mathrm{E}-04)\end{array}$ \\
\hline $\mathrm{X}^{-} \mathrm{SE}(501)$ & 4.5 & $\begin{array}{c}\mathbf{0 . 3} \pm \mathbf{0 . 2} \\
(-1.8 \mathrm{E}-03)\end{array}$ & $\begin{array}{c}\mathbf{1 . 5} \pm \mathbf{0 . 4} \\
(-1.0 \mathrm{E}-03)\end{array}$ & $\begin{array}{l}\mathbf{1 2 . 2} \pm \mathbf{2 . 3} \\
(-7.2 \mathrm{E}-04)\end{array}$ & $\begin{array}{c}\mathbf{2 1 4 . 7} \pm \mathbf{1 3 . 6} \\
(1.6 \mathrm{E}-03)\end{array}$ & $\begin{array}{c}>\mathbf{5 n s} \\
(-7.4 \mathrm{E}-04)\end{array}$ \\
\hline $\mathrm{X}^{-\mathrm{SE}}$ & 5.6 & $\begin{array}{c}\mathbf{0 . 2} \pm \mathbf{0 . 1} \\
(-3.1 \mathrm{E}-03)\end{array}$ & $\begin{array}{c}\mathbf{1 . 4} \pm \mathbf{0 . 3} \\
(-1.3 \mathrm{E}-03)\end{array}$ & $\begin{array}{c}9.9 \pm 1.8 \\
(-7.2 \mathrm{E}-04)\end{array}$ & $\begin{array}{c}\mathbf{1 7 1 . 6} \pm \mathbf{7 . 7} \\
(1.9 \mathrm{E}-03)\end{array}$ & $\begin{array}{c}>\mathbf{5 n s} \\
(-1.0 \mathrm{E}-03)\end{array}$ \\
\hline $\mathrm{X}^{-S E}$ & 7.3 & $\begin{array}{c}\mathbf{0 . 2} \pm \mathbf{0 . 1} \\
(-2.6 \mathrm{E}-03)\end{array}$ & $\begin{array}{c}\mathbf{1 . 3} \pm \mathbf{0 . 3} \\
(-1.7 \mathrm{E}-03)\end{array}$ & $\begin{array}{c}\mathbf{7 . 4} \pm \mathbf{1} \\
(-9.5 \mathrm{E}-04)\end{array}$ & $\begin{array}{c}\mathbf{1 6 8 . 4} \pm \mathbf{4 . 8} \\
(2.5 \mathrm{E}-03)\end{array}$ & $\begin{array}{c}>\mathbf{5 n s} \\
(-1.0 \mathrm{E}-03)\end{array}$ \\
\hline $\mathrm{X}^{-\mathrm{SE}}$ & 9 & $\begin{array}{c}\mathbf{0 . 3} \pm \mathbf{0 . 1} \\
(-2.8 \mathrm{E}-03)\end{array}$ & $\begin{array}{c}\mathbf{1 . 4} \pm \mathbf{0 . 3} \\
(-2.0 \mathrm{E}-03)\end{array}$ & $\begin{array}{c}\mathbf{7} \pm \mathbf{1 . 1} \\
(-1.1 \mathrm{E}-03)\end{array}$ & $\begin{array}{c}\mathbf{1 5 4 . 3} \pm \mathbf{3 . 2} \\
(3.4 \mathrm{E}-03)\end{array}$ & $\begin{array}{c}>\mathbf{5 n s} \\
(-1.1 \mathrm{E}-03)\end{array}$ \\
\hline $\mathrm{X}^{-\mathrm{SE}}$ & 11.3 & $\begin{array}{c}\mathbf{0 . 4} \pm \mathbf{0 . 2} \\
(-2.3 \mathrm{E}-03)\end{array}$ & $\begin{array}{c}\mathbf{1 . 1} \pm \mathbf{0 . 5} \\
(-2.4 \mathrm{E}-03)\end{array}$ & $\begin{array}{r}\mathbf{4 . 2} \pm \mathbf{0 . 6} \\
(-1.9 \mathrm{E}-03)\end{array}$ & $\begin{array}{c}\mathbf{1 4 9 . 1} \pm \mathbf{2 . 7} \\
(4.4 \mathrm{E}-03)\end{array}$ & $\begin{array}{c}>\mathbf{5 n s} \\
(-1.4 \mathrm{E}-03)\end{array}$ \\
\hline $\mathrm{X}-\mathrm{SE}$ & 14.7 & $\begin{array}{c}\mathbf{0 . 4} \pm \mathbf{0 . 1} \\
(-5.8 \mathrm{E}-03)\end{array}$ & $\begin{array}{c}\mathbf{1 . 4} \pm \mathbf{0 . 2} \\
(-5.0 \mathrm{E}-03)\end{array}$ & $\begin{array}{c}\mathbf{7 . 3} \pm \mathbf{1} \\
(-2.2 \mathrm{E}-03)\end{array}$ & $\begin{array}{c}\mathbf{1 5 3 . 9} \pm \mathbf{2 . 5} \\
(6.9 \mathrm{E}-03)\end{array}$ & $\begin{array}{c}>\mathbf{5 n s} \\
(-1.8 \mathrm{E}-03)\end{array}$ \\
\hline$X^{-}$SE & 20.9 & $\begin{array}{c}\mathbf{0 . 4} \pm \mathbf{0 . 1} \\
(-1.1 \mathrm{E}-02)\end{array}$ & $\begin{array}{r}\mathbf{1 . 2} \pm \mathbf{0 . 4} \\
(-7.7 \mathrm{E}-03)\end{array}$ & $\begin{array}{c}\mathbf{4 . 3} \pm \mathbf{0 . 9} \\
(-3.7 \mathrm{E}-03)\end{array}$ & $\begin{array}{c}\mathbf{1 3 2 . 3} \pm \mathbf{1 . 9} \\
(1.2 \mathrm{E}-02)\end{array}$ & $\begin{array}{c}>\mathbf{5 n s} \\
(-2.7 \mathrm{E}-03)\end{array}$ \\
\hline $\mathrm{X}^{-} \mathrm{SE}$ (501) & 33.9 & $\begin{array}{c}\mathbf{0 . 4} \pm \mathbf{0 . 1} \\
(-1.7 \mathrm{E}-02)\end{array}$ & $\begin{array}{c}\mathbf{1 . 3} \pm \mathbf{0 . 5} \\
(-1.2 \mathrm{E}-02)\end{array}$ & $\begin{array}{c}\mathbf{5 . 6} \pm \mathbf{1 . 1} \\
(-5.7 \mathrm{E}-03)\end{array}$ & $\begin{array}{c}\mathbf{1 4 3 . 8} \pm \mathbf{1 . 7} \\
(2.0 \mathrm{E}-02)\end{array}$ & $\begin{array}{c}>\mathbf{5 n s} \\
(-1.9 \mathrm{E}-03)\end{array}$ \\
\hline $\mathrm{X}^{-} \mathrm{SE}$ & 67. & $\begin{array}{c}\mathbf{0 . 3} \pm \mathbf{0 . 1} \\
(-3.4 \mathrm{E}-02)\end{array}$ & $\begin{array}{c}\mathbf{1 . 2} \pm \mathbf{0 . 1} \\
(-2.5 \mathrm{E}-02)\end{array}$ & $\begin{array}{l}\mathbf{1 1 . 5} \pm \mathbf{2 . 3} \\
(-3.5 \mathrm{E}-03)\end{array}$ & $\begin{array}{c}\mathbf{1 3 6 . 6} \pm \mathbf{2 . 1} \\
(3.5 \mathrm{E}-02)\end{array}$ & $\begin{array}{c}>\mathbf{5 n s} \\
(-3.5 \mathrm{E}-03)\end{array}$ \\
\hline
\end{tabular}

Table S5. Time constants and amplitudes of fit components for the dynamics of the exciton bleach (X`SE, $501 \mathrm{~nm}$ ) signal extracted from TA spectra of CdSe NPLs at $85 \mathrm{~K}$. Fitted time constants larger than $5 \mathrm{~ns}$ are not explicitly reported, due to the limited length of our optical track. 


\begin{tabular}{|c|c|c|c|c|c|c|}
\hline Signal (nm) & $<\mathrm{N}\rangle$ & $\begin{array}{l}\tau 1 \mathrm{ps} \\
\text { (A1) }\end{array}$ & $\begin{array}{l}\tau 2 \mathrm{ps} \\
\text { (A2) }\end{array}$ & $\begin{array}{l}\tau 3 \mathrm{ps} \\
\text { (A3) }\end{array}$ & $\begin{array}{l}\tau 4 \mathrm{ps} \\
\text { (A4) }\end{array}$ & $\begin{array}{l}\tau 5 \mathrm{ps} \\
\text { (A5) }\end{array}$ \\
\hline $\mathrm{X}^{-} \mathrm{SE}(502)$ & 2.3 & $\begin{array}{c}\mathbf{0 . 5} \pm \mathbf{0 . 3} \\
(-3.0 \mathrm{E}-04)\end{array}$ & $\begin{array}{c}\mathbf{6 . 4} \pm \mathbf{3 . 2} \\
(-2.3 \mathrm{E}-04)\end{array}$ & -- & $\begin{array}{c}\mathbf{1 9 6 . 8} \pm \mathbf{1 9 . 5} \\
(4.2 \mathrm{E}-04)\end{array}$ & $\begin{array}{c}>\mathbf{5 n s} \\
(-3.4 \mathrm{E}-04)\end{array}$ \\
\hline$X-S E(502)$ & 3.4 & $\begin{array}{c}\mathbf{0 . 4} \pm \mathbf{0 . 2} \\
(-4.3 \mathrm{E}-04)\end{array}$ & $\begin{array}{c}2.3 \pm \mathbf{1 . 1} \\
(-2.4 \mathrm{E}-04)\end{array}$ & $\begin{array}{r}\mathbf{1 3 . 4} \pm \mathbf{5 . 5} \\
(-3.1 \mathrm{E}-04)\end{array}$ & $\begin{array}{c}\mathbf{1 8 7 . 2} \pm \mathbf{2 2 . 7} \\
(7.5 \mathrm{E}-04)\end{array}$ & $\begin{array}{c}>\mathbf{5} \mathbf{~ n s} \\
(-5.0 \mathrm{E}-04)\end{array}$ \\
\hline$X-S E(502)$ & 4.5 & $\begin{array}{c}\mathbf{0 . 2} \pm \mathbf{0 . 1} \\
(-1.5 \mathrm{E}-02)\end{array}$ & $\begin{array}{c}\mathbf{1 . 5} \pm \mathbf{0 . 4} \\
(-9.4 \mathrm{E}-04)\end{array}$ & $\begin{array}{l}11.9 \pm 2.7 \\
(-5.7 \mathrm{E}-04)\end{array}$ & $\begin{array}{c}\mathbf{2 1 0 . 2} \pm \mathbf{1 7 . 4} \\
(1.3 \mathrm{E}-03)\end{array}$ & $\begin{array}{c}>\mathbf{5} \mathbf{~ n s} \\
(-5.5 \mathrm{E}-04)\end{array}$ \\
\hline $\mathrm{X}$ SE (502) & 5.6 & $\begin{array}{c}\mathbf{0 . 2} \pm \mathbf{0 . 1} \\
(-5.5 \mathrm{E}-03)\end{array}$ & $\begin{array}{c}\mathbf{1 . 4} \pm \mathbf{0 . 4} \\
(-9.6 \mathrm{E}-04)\end{array}$ & $\begin{array}{c}\mathbf{6 . 9} \pm \mathbf{1 . 5} \\
(-6.3 \mathrm{E}-04)\end{array}$ & $\begin{array}{c}\mathbf{1 7 3 . 6} \pm \mathbf{8 . 7} \\
(1.5 \mathrm{E}-03)\end{array}$ & $\begin{array}{c}>\mathbf{5} \mathbf{~ n s} \\
(-7.4 \mathrm{E}-04)\end{array}$ \\
\hline $\mathrm{X}^{-} \mathrm{SE}(502)$ & 7.3 & $\begin{array}{c}\mathbf{0 . 2} \pm \mathbf{0 . 1} \\
(-5.6 \mathrm{E}-03)\end{array}$ & $\begin{array}{c}\mathbf{1 . 6} \pm \mathbf{0 . 2} \\
(-1.7 \mathrm{E}-03)\end{array}$ & $\begin{array}{l}15.3 \pm 4.1 \\
(-4.2 \mathrm{E}-04)\end{array}$ & $\begin{array}{c}\mathbf{1 6 2 . 8} \pm \mathbf{8 . 4} \\
(2.0 \mathrm{E}-03)\end{array}$ & $\begin{array}{c}>\mathbf{5} \mathbf{n s} \\
(-8.2 \mathrm{E}-04)\end{array}$ \\
\hline $\mathrm{X}^{-} \mathrm{SE}$ (502) & 9 & $\begin{array}{c}\mathbf{0 . 2} \pm \mathbf{0 . 1} \\
(-1.7 \mathrm{E}-03)\end{array}$ & $\begin{array}{c}\mathbf{1 . 5} \pm \mathbf{0 . 3} \\
(-1.7 \mathrm{E}-03)\end{array}$ & $\begin{array}{l}\mathbf{1 0 . 7} \pm \mathbf{1 . 9} \\
(-7.8 \mathrm{E}-04)\end{array}$ & $\begin{array}{c}\mathbf{1 6 1 . 9} \pm \mathbf{5 . 9} \\
(2.4 \mathrm{E}-03)\end{array}$ & $\begin{array}{c}>\mathbf{5} \mathbf{~ n s} \\
(-7.1 \mathrm{E}-04)\end{array}$ \\
\hline $\mathrm{X}^{-\mathrm{SE}}$ & 11.3 & $\begin{array}{c}\mathbf{0 . 3} \pm \mathbf{0 . 1} \\
(-2.9 \mathrm{E}-03)\end{array}$ & $\begin{array}{c}\mathbf{1 . 2} \pm \mathbf{0 . 3} \\
(-2.4 \mathrm{E}-03)\end{array}$ & $\begin{array}{c}\mathbf{4 . 8} \pm \mathbf{0 . 9} \\
(-1.3 \mathrm{E}-03)\end{array}$ & $\begin{array}{c}\mathbf{1 5 0 . 3} \pm \mathbf{3 . 4} \\
(3.5 \mathrm{E}-03)\end{array}$ & $\begin{array}{c}>\mathbf{5} \mathbf{~ n s} \\
(-9.5 \mathrm{E}-04)\end{array}$ \\
\hline $\mathrm{X}^{-\mathrm{SE}}$ & 14.7 & $\begin{array}{c}\mathbf{0 . 3} \pm \mathbf{0 . 1} \\
(-5.6 \mathrm{E}-03)\end{array}$ & $\begin{array}{c}\mathbf{1 . 4} \pm \mathbf{0 . 3} \\
(-4.3 \mathrm{E}-03)\end{array}$ & $\begin{array}{c}6.9 \pm 6.9 \\
(-2.0 \mathrm{E}-03)\end{array}$ & $\begin{array}{c}155.4 \pm 1.5 \\
(5.5 \mathrm{E}-03)\end{array}$ & $\begin{array}{c}>\mathbf{5} \mathbf{~ n s} \\
(-1.2 \mathrm{E}-03)\end{array}$ \\
\hline $\mathrm{X}^{-\mathrm{SE}}$ & 20.9 & $\begin{array}{c}\mathbf{0 . 3} \pm \mathbf{0 . 1} \\
(-1.1 \mathrm{E}-02)\end{array}$ & $\begin{array}{c}\mathbf{1 . 2} \pm \mathbf{0 . 5} \\
(-6.2 \mathrm{E}-03)\end{array}$ & $\begin{array}{c}4.2 \pm 1 \\
(-3.0 \mathrm{E}-03)\end{array}$ & $\begin{array}{c}\mathbf{1 3 2 . 5} \pm \mathbf{2 . 1} \\
(9.7 \mathrm{E}-03)\end{array}$ & $\begin{array}{c}>\mathbf{5} \mathbf{~ n s} \\
(-1.8 \mathrm{E}-03)\end{array}$ \\
\hline $\mathrm{X}$ SE (502) & 33.9 & $\begin{array}{c}\mathbf{0 . 4} \pm \mathbf{0 . 1} \\
(-1.8 \mathrm{E}-02)\end{array}$ & $\begin{array}{c}\mathbf{1 . 9} \pm \mathbf{0 . 5} \\
(-7.8 \mathrm{E}-03)\end{array}$ & $\begin{array}{c}7.2 \pm \mathbf{2} \\
(-3.0 \mathrm{E}-03)\end{array}$ & $\begin{array}{c}\mathbf{1 4 5 . 9} \pm \mathbf{2 . 3} \\
(1.6 \mathrm{E}-02)\end{array}$ & $\begin{array}{c}>\mathbf{5} \mathbf{~ n s} \\
(-1.2 \mathrm{E}-03)\end{array}$ \\
\hline $\mathrm{X}^{-} \mathrm{SE}$ (502) & 67. & $\begin{array}{c}\mathbf{0 . 3} \pm \mathbf{0 . 1} \\
(-3.5 \mathrm{E}-02)\end{array}$ & $\begin{array}{c}1.2 \pm \mathbf{0 . 2} \\
(-2.1 \mathrm{E}-02)\end{array}$ & $\begin{array}{l}\mathbf{1 1 . 5} \pm \mathbf{3 . 3} \\
(-1.8 \mathrm{E}-03)\end{array}$ & $\begin{array}{c}\mathbf{1 3 6 . 5} \pm \mathbf{2 . 1} \\
(2.8 \mathrm{E}-02)\end{array}$ & $\begin{array}{c}>\mathbf{5} \mathbf{~ n s} \\
(-2.6 \mathrm{E}-03)\end{array}$ \\
\hline
\end{tabular}

Table S6. Time constants and amplitudes of fit components for the dynamics of the exciton bleach (X`SE, $504 \mathrm{~nm}$ ) signal extracted from TA spectra of CdSe NPLs at $85 \mathrm{~K}$. Fitted time constants larger than $5 \mathrm{~ns}$ are not explicitly reported, due to the limited length of our optical track. 


\section{References}

1. Li, Q.; Lian, T. Area- and Thickness-Dependent Biexciton Auger Recombination in Colloidal CdSe Nanoplatelets: Breaking the "Universal Volume Scaling Law". Nano Lett. 2017, 17, (5), 3152-3158.

2. McPhail, M. R.; Campbell, G. P.; Bedzyk, M. J.; Weiss, E. A. Structural Features of PbS Nanocube Monolayers upon Treatment with Mono- and Dicarboxylic Acids and Thiols at a Liquid-Air Interface. Langmuir 2016, 32, (26), 6666-6673.

3. Antolinez, F. V.; Rabouw, F. T.; Rossinelli, A. A.; Keitel, R. C.; Cocina, A.; Becker, M. A.; Norris, D. J. Trion Emission Dominates the Low-Temperature Photoluminescence of CdSe Nanoplatelets. Nano Lett. 2020, 20, (8), 5814-5820.

4. $\quad$ Irgen-Gioro, S.; Wu, Y.; López-Arteaga, R.; Padgaonkar, S.; Olding, J. N.; Weiss, E. A. Evidence for Two Time Scale-Specific Blinking Mechanisms in Room-Temperature Single Nanoplatelets. J. Phys. Chem. C 2021, 125, (24), 13485-13492.

5. $\quad$ Ayari, S.; Quick, M. T.; Owschimikow, N.; Christodoulou, S.; Bertrand, G. H. V.; Artemyev, M.; Moreels, I.; Woggon, U.; Jaziri, S.; Achtstein, A. W. Tuning trion binding energy and oscillator strength in a laterally finite 2D system: CdSe nanoplatelets as a model system for trion properties. Nanoscale 2020, 12, (27), 14448-14458.

6. McArthur, E. A.; Morris-Cohen, A. J.; Knowles, K. E.; Weiss, E. A. Charge Carrier Resolved Relaxation of the First Excitonic State in CdSe Quantum Dots Probed with NearInfrared Transient Absorption Spectroscopy. J. Phys. Chem. B 2010, 114, (45), 14514-14520.

7. Li, Q.; Liu, Q.; Schaller, R. D.; Lian, T. Reducing the Optical Gain Threshold in TwoDimensional CdSe Nanoplatelets by the Giant Oscillator Strength Transition Effect. J. Phys. Chem. Lett. 2019, 10, (7), 1624-1632.

8. $\quad$ Yeltik, A.; Delikanli, S.; Olutas, M.; Kelestemur, Y.; Guzelturk, B.; Demir, H. V. Experimental Determination of the Absorption Cross-Section and Molar Extinction Coefficient of Colloidal CdSe Nanoplatelets. J. Phys. Chem. C 2015, 119, (47), 26768-26775.

9. Brumberg, A.; Kirschner, M. S.; Diroll, B. T.; Williams, K. R.; Flanders, N. C.; Harvey, S. M.; Leonard, A. A.; Watkins, N. E.; Liu, C.; Kinigstein, E. D.; Yu, J.; Evans, A. M.; Liu, Y.; Cuthriell, S. A.; Panuganti, S.; Dichtel, W. R.; Kanatzidis, M. G.; Wasielewski, M. R.; Zhang, X.; Chen, L. X.; Schaller, R. D. Anisotropic Transient Disordering of Colloidal, TwoDimensional CdSe Nanoplatelets upon Optical Excitation. Nano Lett. 2021, 21, (3), 1288-1294. 10. Geiregat, P.; Tomar, R.; Chen, K.; Singh, S.; Hodgkiss, J. M.; Hens, Z. Thermodynamic Equilibrium between Excitons and Excitonic Molecules Dictates Optical Gain in Colloidal CdSe Quantum Wells. J. Phys. Chem. Lett. 2019, 10, (13), 3637-3644.

11. Shornikova, E. V.; Biadala, L.; Yakovlev, D. R.; Sapega, V. F.; Kusrayev, Y. G.; Mitioglu, A. A.; Ballottin, M. V.; Christianen, P. C. M.; Belykh, V. V.; Kochiev, M. V.; Sibeldin, N. N.; Golovatenko, A. A.; Rodina, A. V.; Gippius, N. A.; Kuntzmann, A.; Jiang, Y.; Nasilowski, M.; Dubertret, B.; Bayer, M. Addressing the exciton fine structure in colloidal nanocrystals: the case of CdSe nanoplatelets. Nanoscale 2018, 10, (2), 646-656.

12. Shornikova, E. V.; Yakovlev, D. R.; Biadala, L.; Crooker, S. A.; Belykh, V. V.; Kochiev, M. V.; Kuntzmann, A.; Nasilowski, M.; Dubertret, B.; Bayer, M. Negatively Charged Excitons in CdSe Nanoplatelets. Nano Lett. 2020, 20, (2), 1370-1377.

13. Biadala, L.; Liu, F.; Tessier, M. D.; Yakovlev, D. R.; Dubertret, B.; Bayer, M. Recombination Dynamics of Band Edge Excitons in Quasi-Two-Dimensional CdSe Nanoplatelets. Nano Lett. 2014, 14, (3), 1134-1139. 